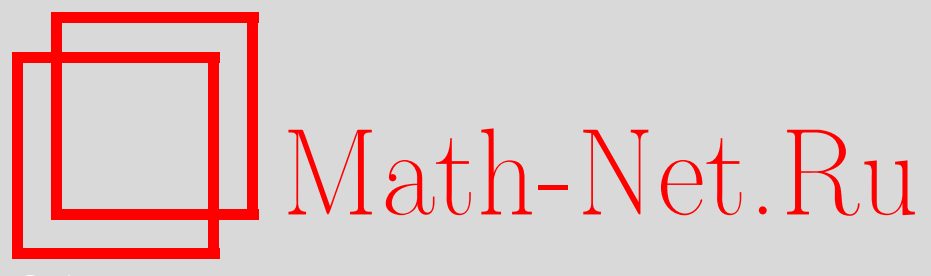

Р. М. Тригуб, Приближение периодических функций тригонометрическими полиномами с эрмитовой интерполяцией и учетом положения точки, Изв. РАН. Сер. матем., 2009, том 73, выпуск 4, 49-76

DOI: https://doi.org/10.4213/im2722

Использование Общероссийского математического портала Math-Net.Ru подразумевает, что вы прочитали и согласны с пользовательским соглашением http://www . mathnet.ru/rus/agreement

Параметры загрузки:

IP : 54.162 .27 .143

26 апреля 2023 г., 10:36:14

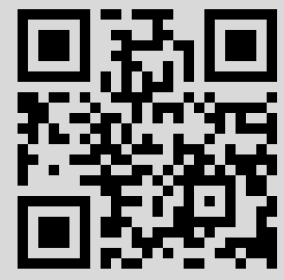




\title{
Р. М. Тригуб \\ Приближение периодических функций тригонометрическими полиномами с эрмитовой интерполяцией и учетом положения точки
}

\begin{abstract}
Доказана общая прямая теорема об одновременном приближении гладких периодических функций и их производных тригонометрическими полиномами и их производными с эрмитовой интерполяцией и учетом положения точки. Исследован порядок аппроксимации полиномами, имеющими графики, расположенные выше и ниже графика функции на заданных интервалах. Доказано несколько неравенств для эрмитовой интерполяции с абсолютными константами (при любой системе узлов). Впервые получена теорема о приближении функций полиномами порядка наилучшего приближения при интерполяции в заданной системе узлов. Кроме того, указан способ построения эрмитовых интерполяционных тригонометрических полиномов для периодических функций (в случае одного узла - это тригонометрический полином Тейлора).

Библиография: 15 наименований.
\end{abstract}

Ключевые слова: тригонометрический полином Тейлора, наилучшее приближение, модуль гладкости, двусторонние оценки приближения, кусочно односторонняя аппроксимация, факторизация дифференциальных операторов.

\section{§ 1. Введение. Формулировка результатов}

Пусть $\mathbb{T}=[-\pi, \pi], C^{r}(\mathbb{T}), r \in \mathbb{Z}_{+},-$множество $2 \pi$-периодических функций из $C^{r}(\mathbb{R}),\|f\|=\max _{x \in \mathbb{T}}|f(x)|$ и $\tau(x)=\sum_{k=p}^{n} c_{k} e^{i k x}-$ тригонометрический полином со спектром на $[p, n], p \leqslant n$; при $n=-p \in \mathbb{Z}_{+}$это полином порядка не выше $n$. Через $\gamma(a, b, \ldots, w)$ будем обозначать положительные величины, зависящие лишь от $a, b, \ldots, w$.

Пусть даны узлы интерполяции $x_{1}<x_{2}<\cdots<x_{m}<x_{1}+2 \pi=x_{m+1}$, $\Pi=\left\{x_{s}\right\}$.

Рассмотрим сначала случай $m=1$ и $x_{1}=0$. Построим полином наименьшего порядка с условием $\tau^{(\nu)}(0)=f^{(\nu)}(0), 0 \leqslant \nu \leqslant r$ (тригонометрический полином Тейлора). Искомый эрмитов полином естественно искать в виде

$$
\sum_{k=0}^{r} f^{(k)}(0) h_{k, r}(x),
$$

где $h_{k, r}-$ полином порядка не выше $\frac{r+1}{2}$, удовлетворяющий условию

$$
h_{k, r}(x)=\frac{x^{k}}{k !}+O\left(x^{r+1}\right), \quad x \rightarrow 0 .
$$


При четном $r$ полином $h_{k, r}$ определяется однозначно (явные формулы см. ниже в лемме 1$)$, при нечетном $r$ полагаем $h_{0, r} \equiv 1$, а при $k \geqslant 1$ имеем $h_{k, r}=h_{k+1, r+1}^{\prime}$.

Теорема 1. Справедливы следующие утверждения:

а) для любой функиии $f \in C^{2 n}(\mathbb{T}), n \in \mathbb{N}$, и для любого $x \in\left[-\frac{\pi}{n}, \frac{\pi}{n}\right]$ сущеcтвует $\theta \in[0,1]$ такое, что

$$
f(x)=\sum_{k=0}^{2 n} f^{(k)}(0) h_{k, 2 n}(x)+\left[D_{2 n} f(\theta x)-D_{2 n} f(0)\right] h_{2 n, 2 n}(x),
$$

где $D_{2 n} f(x)=q_{n}\left(\frac{d^{2}}{d x^{2}}\right) f(x) u q_{n}(x)=\Pi_{k=1}^{n}\left(x+k^{2}\right)$;

b) для любой функции $f \in C^{r}(\mathbb{T}), r \in \mathbb{N}$, существует тригонометрический полином $\tau_{f}$ порядка не выше $\frac{r+1}{2}$ такой, что для $\nu \in[0, r]$ и всех $x \in \mathbb{R}$ выполнено неравенство

$$
\left|f^{(\nu)}(x)-\tau_{f}^{(\nu)}(x)\right| \leqslant \gamma(r) \omega\left(f^{(r)} ; \pi\right)\left|\sin \frac{x}{2}\right|^{r-\nu},
$$

где $\omega-$ модуль непрерывности.

Зная способ построения эрмитова полинома для одного узла, можно найти эрмитов полином для любого числа узлов (см. рассуждение, приведенное перед леммой 2).

В теории аппроксимации хорошо известны теоремы о порядке, который определяется модулем гладкости $\omega_{k}$ функции или ее производной, приближения гладких функций полиномами растущих порядков (см., например, [1, гл. IV] или $[2$, гл. IV]). В работе [3] получена общая теорема о приближении функций на отрезке вещественной оси алгебраическими полиномами с эрмитовой интерполяцией в заданных узлах, а в работе [4] изучалась и односторонняя аппроксимация при дополнительных условиях, налагаемых на функцию.

Теорема 2. Для любой функиии $f \in C^{r}(\mathbb{T}), r \in \mathbb{Z}_{+}$, и любой системы узлов $\Pi=\left\{x_{s}\right\}$ при любом $k \in \mathbb{N}$ u $n \geqslant\left[\frac{m(r+1)}{2}\right]$ (здесь $[\cdot]$ - иелая часть числа) существует полином $\tau_{n}$ порядка не выше $n$ такой, что при $\nu \in[0, r]$ и всех $x \in \mathbb{R}$ выполнено неравенство

$$
\left|f^{(\nu)}(x)-\tau_{n}^{(\nu)}(x)\right| \leqslant \gamma(r, k, \Pi) \delta_{n}^{r-\nu}(x) \omega_{k}\left(f^{(r)} ; \frac{1}{n}\left(n \delta_{n}(x)\right)^{\frac{1}{k}}\right),
$$

¿де

$$
\delta_{n}(x)=\min \left\{\frac{1}{n},\left|\sin \frac{x-x_{s}}{2}\right|, 1 \leqslant s \leqslant m\right\} .
$$

Показатель степени $\frac{1}{k}$ увеличить нельзя.

Подобная оценка приближения функций на отрезке алгебраическими полиномами наименьшей степени получена в работе [5], в случае полиномов любой степени и двух узлов интерполяции в концах отрезка - в [6], а в общем случае B [3].

В теореме 2, кроме прочего, найден наименьший порядок полиномов, которые осуществляют указанное приближение. Поэтому константа $\gamma$ зависит, 
конечно, от системы узлов $\left\{x_{s}\right\}$, точнее от $\min _{s}\left|x_{s}-x_{s+1}\right|$. При изучении комонотонных аппроксимаций появились результаты о порядке приближения с константами, зависящими лишь от числа $m$ узлов (см., например, [7]). Иногда при достаточно больших $n$ можно поставить в соответствующих неравенствах, как оказалось, абсолютные константы (см. ниже теорему 3). Заметим, что в случае равноотстоящих $m$ узлов еще Д. Джексон построил такие интерполяционные полиномы порядка $2 m$, а С. Н. Бернштейн уменьшил порядок до $m(1+\varepsilon)$ при любом $\varepsilon>0$ (см., например, [8, гл. 10]).

ТЕОрема 3. Справедливы следующие утверждения:

а) существует абсолютная константа с такая, что для любого $r \in \mathbb{Z}_{+} u$ для любых наборов $\left\{x_{s}\right\},\left\{y_{s, \nu}\right\}, 1 \leqslant s \leqslant m, 0 \leqslant \nu \leqslant r$, можно построить тригонометрический полином $\tau$ любого достаточно большого порядка, зависящего от $r$ u $\min \left|x_{s}-x_{s+1}\right|$, который удовлетворяет условиям

$$
\tau^{(\nu)}\left(x_{s}\right)=y_{s, \nu}, \quad 1 \leqslant s \leqslant m, \quad 0 \leqslant \nu \leqslant r, \quad\|\tau\| \leqslant c \max _{s, \nu}\left|y_{s, \nu}\right|
$$

b) для любых узлов $\Pi=\left\{x_{s}\right\}$ u $\varepsilon>0$ существует иисло $N=N(\varepsilon, \Pi)$ такое, что при любом $n \geqslant N$ для любой функиии $f \in C(\mathbb{T})$ можно построить полином $\tau_{n}$ порядка не выше $n$, удовлетворяющий условиям $\tau_{n}\left(x_{s}\right)=f\left(x_{s}\right)$, $1 \leqslant s \leqslant m, u$

$$
\left\|f-\tau_{n}\right\| \leqslant(2+\varepsilon) E_{n}^{T}(f),
$$

где $E_{n}^{T}(f)$-наилучшее приближение $f$ тригонометрическими полиномами порядка не выше $n$ в пространстве $C(\mathbb{T})$. При этом множитель $(2+\varepsilon)$ нельзя заменить на $(2-\varepsilon)$ ни при одном $n$.

Отсюда следует, что в случае равноотстоящих узлов, когда, например, $x_{s}=$ $\frac{2 s \pi}{2 m+1}, 0 \leqslant s \leqslant 2 m$, и лагранжевой интерполяции $(r=0)$ можно брать полиномы порядка $n \geqslant N=c m \log (m+1)$, и порядок приближения будет наилучшим порядком с абсолютной константой.

Рассмотрим теперь односторонние приближения, когда график полинома находится выше (или ниже) графика функции. Прямые теоремы о таких приближениях уже довольно давно успешно применены к доказательству тауберовых теорем с остаточным членом (см., например, [9, гл. I]).

Кусочно односторонняя аппроксимация не исследована, а из нее следует, например, копозитивная аппроксимация, когда знак приближающего полинома совпадает со знаком функции на конечном числе интервалов. Здесь функция предполагается, конечно, вещественной, а знаки для $f-\tau_{n}$ чередующимися. Отметим, что не всегда существует полином (и, вообще, гладкая функция) с графиком, находящимся выше графика непрерывной функции, который должен еще совпадать с функцией в данной точке. Так, для функции $f(x)=\sqrt{|x|} \operatorname{sign} x$ нет полинома с графиком, находящимся всюду выше или ниже графика функции при условии интерполяции в нуле, а переход в нуле с одной стороны графика на другую возможен лишь в одном из двух вариантов (см. критерий в лемме 11). Поэтому в следующей теореме предполагается дифференцируемость функции. В силу периодичности функции и полинома число $m$ узлов интерполяции (перемены знака) должно быть четным. 
Tеорема 4. Пусть $f \in C^{1}(\mathbb{T}), k \in \mathbb{N},\left\{x_{s}\right\}_{s=1}^{m}$ - узльь и число $m$ четное. Для любого $n \geqslant \frac{m}{2}$ существует полином $\tau_{n}$ порядка не выше $n$ такой, что при $x \in\left[x_{s}, x_{s+1}\right], s \in[1, m], u \delta_{n}$ из теоремы 2 имеем

$$
\omega_{k}\left(f^{\prime} ; \frac{1}{n}\right) \delta_{n}(x) \leqslant(-1)^{s}\left(f(x)-\tau_{n}(x)\right) \leqslant \gamma(k, \Pi) \omega_{k}\left(f^{\prime} ; \frac{1}{n}\right) \delta_{n}(x) .
$$

Доказательства теорем 1-4 приводятся ниже, в § 3. Отметим также, что оператор $\tau_{n}$ в теореме 4 не является линейным. В случае функций, заданных на отрезке, и алгебраических полиномов можно построить линейные операторы одностороннего приближения при некоторых дополнительных условиях, налагаемых на функцию [3]. Основная идея построения нелинейного оператора одностороннего приближения состоит в добавлении к полиному "хорошего" приближения специального полинома (лемма 10, случай алгебраических полиномов см. в [10]).

Основные результаты настоящей статьи анонсированы в [11].

\section{§ 2. Вспомогательные утверждения}

Спектром spec $f$ функции $f \in L(\mathbb{T})$ называют множество номеров ее коэффициентов Фурье $\hat{f}(k)$, которые не равны нулю. При сдвиге, т. е. при замене $x$ на $x+h$, спектр функции не меняется, а при умножении функции на $e^{i q x}, q \in \mathbb{Z}$, происходит сдвиг спектра на $q$.

Ближе всего к алгебраическим полиномам находятся тригонометрические полиномы степенного типа (спектр на $\mathbb{Z}_{+}$). Достаточно сделать замену $e^{i x}=z$. Например, лагранжев интерполяционный полином для системы узлов $\left\{x_{s}\right\}$ : $x_{1}<x_{2}<\cdots<x_{m}<x_{m+1}=x_{1}+2 \pi$, определяемый условиями $\tau\left(x_{s}\right)=y_{s}$, $1 \leqslant s \leqslant m, \operatorname{spec} \tau \subset[0, m-1]$, равен

$$
\sum_{s=1}^{m} y_{s} \frac{\prod_{k \neq s}\left(e^{i x}-e^{i x_{k}}\right)}{\prod_{k \neq s}\left(e^{i x_{s}}-e^{i x_{k}}\right)}
$$

Приведем простые свойства полиномов, которые используются далее.

I. Полином $\tau(z)=\sum_{k=p}^{n} \hat{\tau}(k) e^{i k z}$ с условиями $n \geqslant p$ и $\hat{\tau}(p) \hat{\tau}(n) \neq 0$ имеет в полосе $\operatorname{Re} z \in(a, a+2 \pi], a \in \mathbb{R}$, ровно $n-p$ нулей (с учетом кратностей).

II. Для любого набора различных точек $\left\{z_{\nu}\right\}, 1 \leqslant \nu \leqslant k$, из указанной в утверждении I полосы и натуральных чисел $\left\{r_{\nu}\right\}, 1 \leqslant \nu \leqslant k$, с условием $\sum_{\nu=1}^{k} r_{\nu}=n-p+1$, а также для любого набора комплексных чисел $\{y(s)\}$, $0 \leqslant s \leqslant n-p$, существует единственный полином $\tau$ со спектром в $[p, n]$, удовлетворяющий условиям

$$
\tau^{(s)}\left(z_{\nu}\right)=y\left(\sum_{q=0}^{\nu-1} r_{q}+s\right), \quad 1 \leqslant \nu \leqslant k, \quad 0 \leqslant s \leqslant r_{\nu}-1 .
$$

Для того чтобы при вещественных $\left\{z_{\nu}\right\}$ и $\{y(s)\}$ соответствующий полином принимал на $\mathbb{R}$ лишь вещественные значения, спектр его должен быть симметричным относительно нуля $(p=-n)$. 
III. Пусть $r \in \mathbb{Z}_{+}, q \in \mathbb{Z}$. Если полином $\tau$ удовлетворяет условиям $\tau^{(\nu)}\left(x_{s}\right)=$ $f^{(\nu)}\left(x_{s}\right), 0 \leqslant \nu \leqslant r, 1 \leqslant s \leqslant m$, а $g(x)=e^{i q x} f(x)$, то полином $t(x)=e^{i q x} \tau(x)$ удовлетворяет условиям $t^{(\nu)}\left(x_{s}\right)=g^{(\nu)}\left(x_{s}\right), 0 \leqslant \nu \leqslant r, 1 \leqslant s \leqslant m$.

IV. Пусть $\operatorname{spec} \tau \subset[p, n]$ и $\hat{\tau}(n) \neq 0$, a $\operatorname{spec} t \subset[k, m]$ и $\hat{t}(m) \neq 0$. Тогда при $n-p \geqslant m-k \geqslant 0$ имеем

$$
\tau(x)=a(x) t(x)+b(x)
$$

где $a$ и $b-$ полиномы со спектрами соответственно в $[p-k, n-m]$ и $[p, p+m-$ $k-1]$ (полином со спектром в $[p, p-1]$ считается равным нулю тождественно). $\mathrm{B}$ частности, при $p=-n$ и $k=-m, m \leqslant n$, имеем

$$
\tau(x)=a(x) t(x)+e^{i(m-n) x} b(x),
$$

где $\operatorname{spec} a \in[m-n, n-m]$ и $\operatorname{spec} b \in[-m, m-1]$.

Исследуем теперь вопрос: как найти эрмитов интерполяционный полином $\tau_{f, n}$, определяемый условиями $\tau_{f, n}^{(\nu)}\left(x_{s}\right)=f^{(\nu)}\left(x_{s}\right), 0 \leqslant \nu \leqslant r, 1 \leqslant s \leqslant m$ ?

Рассмотрим сначала случай $m=1$ и $x_{1}=0$ (полином Тейлора) при четном $r=2 n$. В силу свойства II полином $h_{k, 2 n}$ порядка не выше $n$ условием (1), т. е. по производным $h_{k, 2 n}^{(\nu)}(0), 0 \leqslant \nu \leqslant 2 n$, определяется однозначно. Поэтому

$$
h_{k, 2 n}(-x)=(-1)^{k} h_{k, 2 n}(x), \quad h_{2 k-1,2 n}(x)=h_{2 k, 2 n}^{\prime}(x) .
$$

Кроме того, легко видеть, что

$$
h_{0,2 n}(x) \equiv 1, \quad h_{2 n, 2 n}(x)=\frac{2^{n}}{(2 n) !}(1-\cos x)^{n},
$$

и при $k \in \mathbb{N}$ имеем

$$
h_{2 k-1,2 n}^{\prime}(x)=h_{2 k-2,2 n}(x)-c_{k, n} h_{2 n, 2 n}(x),
$$

где числовой множитель $c_{k, n}$ определяется однозначно, если учесть, что интеграл от $h^{\prime}$ по периоду равен нулю. Это рекуррентные формулы: по $h_{2 n, 2 n}$ можно найти $h_{2 n-1,2 n}, h_{2 n-2,2 n}$ и т. д.

Приведем явные формулы для $h_{k, 2 n}$.

ЛЕмма 1. Справедливы следующие утверждения:

a) если $q_{n}(x)=\prod_{\nu=1}^{n}\left(x+\nu^{2}\right)=\sum_{s=0}^{n} a_{s, n} x^{s}, m o$

$$
h_{k, 2 n}(x)=\sum_{\left[\frac{k+1}{2}\right] \leqslant s \leqslant n} a_{s, n} h_{2 n, 2 n}^{(2 s-k)}(x)
$$

b) имеем $h_{2 k-1,2 n}(x)=h_{2 k, 2 n}^{\prime}(x)$, a npu $0 \leqslant k \leqslant n$

$$
h_{2 k, 2 n}(x)=\sum_{s=k}^{n} b_{s, k}(1-\cos x)^{s}, \quad b_{s, k}=\frac{2^{2 k-s}}{(2 k) !(2 s) !} \frac{d^{2 s}}{d x^{2 s}}\left\{(\arcsin x)^{2 k}\right\}_{x=0} .
$$


ДокАЗАТЕЛЬство. а) Поскольку $a_{n, n}=1$, то $h_{2 n, 2 n}-$ тот же полином. Проверим, что $h_{k, 2 n}$ удовлетворяет условию (1).

Имеем

$$
h_{k, 2 n}^{\prime}(x)=\sum_{s=\left[\frac{k+1}{2}\right]}^{n} a_{s, n} h_{2 n, 2 n}^{(2 s-k+1)}(x),
$$

откуда $h_{k, 2 n}^{\prime}(x)=h_{k-1,2 n}(x), 1 \leqslant k \leqslant 2 n$, при четном $k$, а при нечетном $k$

$h_{k, 2 n}^{\prime}(x)=\sum_{s=\left[\frac{k}{2}\right]}^{n} a_{s, n} h_{2 n, 2 n}^{(2 s-k+1)}(x)-a_{\frac{k-1}{2}, n} h_{2 n, 2 n}(x)=h_{k-1,2 n}(x)-a_{\frac{k-1}{2}, n} h_{2 n, 2 n}(x)$.

Найдем по указанной формуле $h_{0,2 n}$.

Поскольку оператор $\frac{d^{2}}{d x^{2}}+n^{2}$ понижает порядок $n$ полинома $\tau_{n}$ и умножает свободный член $\hat{\tau}_{n}(0)$ на $n^{2}$, то

$$
h_{0,2 n}(x)=\sum_{s=0}^{n} a_{s, n} h_{2 n, 2 n}^{(2 s)}(x)=\prod_{\nu=1}^{n}\left(\frac{d^{2}}{d x^{2}}+\nu^{2}\right) h_{2 n, 2 n}(x)=(n !)^{2} \hat{h}_{2 n, 2 n}(0) .
$$

Однако

$$
\hat{h}_{2 n, 2 n}(0)=\frac{1}{2 \pi} \int_{-\pi}^{\pi} h_{2 n, 2 n}(x) d x=\frac{1}{2 \pi} \frac{4^{n+1}}{(2 n) !} \int_{0}^{\frac{\pi}{2}} \sin ^{2 n} u d u=\frac{1}{(n !)^{2}},
$$

так что $h_{0,2 n}(x) \equiv 1$. Теперь индукцией по $k$ (от $k-1$ к $k$ )легко получить

$$
h_{k, 2 n}(x)=\frac{x^{k}}{k !}+O\left(x^{2 n+1+\frac{1+(-1)^{k}}{2}}\right), \quad x \rightarrow 0, \quad 0 \leqslant k \leqslant 2 n .
$$

b) Любой четный тригонометрический полином можно представить в указанном виде. Нужно только определить коэффициенты $b_{s, k}$.

После замены $\sin \frac{x}{2}=y$ при $y \rightarrow 0$ полином $h_{2 k, 2 n}$ должен вести себя следующим образом:

$$
\sum_{s=k}^{n} b_{s, k} \cdot 2^{s} y^{2 s}=\frac{(2 \arcsin y)^{2 k}}{(2 k) !}+O\left(y^{2 n+2}\right) .
$$

Это возможно лишь в случае

$$
b_{s, k} \cdot 2^{s}(2 s) !=\frac{4^{k}}{(2 k) !} \frac{d^{2 s}}{d y^{2 s}}\left\{(\arcsin y)^{2 k}\right\}_{y=0}, \quad k \leqslant s \leqslant n,
$$

что и требовалось доказать.

Отметим, что формула из утверждения а) леммы 1 получена при участии В. П. Заставного.

Приведем некоторые свойства полиномов $h_{k, 2 n}$, которые следуют (см. ниже) из леммы 1:

$\alpha)$ для любых $k \in[1,2 n]$ и $x \in[0, \pi]$

$$
h_{k, 2 n}(x) \leqslant \frac{x^{k}}{k !} \leqslant \frac{\pi^{k}}{k !}\left(\sin \frac{x}{2}\right)^{k}, \quad h_{k+1,2 n}^{\prime \prime}(x) \leqslant h_{k, 2 n}^{\prime}(x) \leqslant h_{k-1,2 n}(x) ;
$$


$\beta)$ для любых $k \in[1, n]$ и $x \in[0, \pi]$

$$
\frac{\left(2 \sin \frac{x}{2}\right)^{2 k}}{(2 k) !} \leqslant h_{2 k, 2 n}(x), \quad \frac{\left(2 \sin \frac{x}{2}\right)^{2 k-1}}{(2 k-1) !} \cos \frac{x}{2} \leqslant h_{2 k-1,2 n}(x)
$$

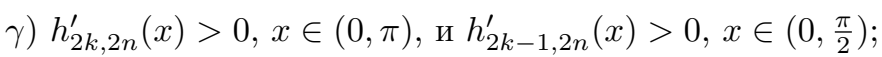

$\delta) h_{2 k, 2 n}(x+\pi)$ - полином с положительными коэффициентами.

Для доказательства этих свойств заметим, что $a_{s, n}>0$ при $0 \leqslant s \leqslant n$. Проверим, что и коэффициенты $b_{s, k}, 0 \leqslant k \leqslant s \leqslant n$, положительны. Биномиальный степенной ряд $\left(1-y^{2}\right)^{-\frac{1}{2}}$ имеет при четных степенях $y$ положительные коэффициенты. Следовательно, и проинтегрированный ряд для $\arcsin y$ имеет положительные коэффициенты. K тому же в формуле из утверждения b) леммы 1 при переходе от $n$ к $n+1$ добавляется лишь одно слагаемое.

Утверждение $\alpha$ ) следует теперь из рекуррентной формулы индукцией по $k$ с учетом того, что $h_{2 n, 2 n}(x)>0$ на $(0,2 \pi)$, а $h_{2 n, 2 n}^{\prime}(x)>0$ на $(0, \pi)$.

Для доказательства $\beta$ ) и $\gamma$ ) нужно в формуле утверждения b) леммы 1 оставить только первое слагаемое для $h_{2 k, 2 n}$ и учесть, что $h_{2 k-1,2 n}=h_{2 k, 2 n}^{\prime}(x)$, $b_{k, k}=\frac{2^{k}}{(2 k) !}$ и $h_{2 n, 2 n}^{\prime \prime}(x)>0$ на $\left(0, \frac{\pi}{2}\right]$.

Утверждение $\delta$ ) следует из утверждения а) или b) леммы 1 и разложения

$$
h_{2 n, 2 n}(x)=\frac{4^{n}}{(2 n) !}\left(\frac{e^{i \frac{x}{2}}-e^{-i \frac{x}{2}}}{2 i}\right)^{2 n}=\frac{1}{(2 n) !} \sum_{\nu=-n}^{n}(-1)^{\nu} \frac{(2 n) !}{(n+\nu) !(n-\nu) !} e^{i \nu x} .
$$

Используя найденную выше формулу Тейлора (эрмитов полином для одного узла), можно построить эрмитов полином для функции с любым числом узлов $\left\{x_{s}\right\}: \tau_{f, r}^{(\nu)}\left(x_{s}\right)=f^{(\nu)}\left(x_{s}\right), 0 \leqslant \nu \leqslant r_{s}, 1 \leqslant s \leqslant m$.

Пусть $t_{1}$ - нетривиальный полином наименьшего порядка, удовлетворяющий условиям $t_{1}^{(\nu)}\left(x_{1}\right)=0,0 \leqslant \nu \leqslant r_{1}$. Полагаем

$$
f_{1}(x)=\frac{f(x)-\sum_{k=0}^{r_{1}} f^{(k)}\left(x_{1}\right) h_{k, r_{1}}\left(x-x_{1}\right)}{t_{1}(x)} .
$$

Тогда полином

$$
\sum_{k=0}^{r_{1}} f^{(k)}\left(x_{1}\right) h_{k, r_{1}}\left(x-x_{1}\right)+t_{1}(x) \sum_{k=0}^{r_{2}} f^{(k)}\left(x_{2}\right) h_{k, r_{2}}\left(x-x_{2}\right)
$$

является эрмитовым для $f$ в двух узлах $x_{1}$ и $x_{2}$. Если $f_{2}$ - частное от деления разности между функцией $f$ и этим полиномом на нетривиальный полином $t_{2}$ наименьшего порядка, удовлетворяющий условиям

$$
t_{2}^{(\nu)}\left(x_{1}\right)=0, \quad 0 \leqslant \nu \leqslant r_{1}, \quad t_{2}^{(\nu)}\left(x_{2}\right)=0, \quad 0 \leqslant \nu \leqslant r_{2},
$$

то полином

$$
\begin{gathered}
\sum_{k=0}^{r_{1}} f^{(k)}\left(x_{1}\right) h_{k, r_{1}}\left(x-x_{1}\right)+t_{1}(x) \sum_{k=0}^{r_{2}} f^{(k)}\left(x_{2}\right) h_{k, r_{2}}\left(x-x_{2}\right) \\
+t_{2}(x) \sum_{k=0}^{r_{3}} f^{(k)}\left(x_{3}\right) h_{k, r_{3}}\left(x-x_{3}\right)
\end{gathered}
$$

является эрмитовым для функции $f$ в трех узлах $x_{1}, x_{2}$ и $x_{3}$. И так далее. 
Считаем для простоты, что $r_{1}=r_{2}=\cdots=r$. Если еще учесть, что при любых $q \in \mathbb{Z}_{+}$и $k \in\left[0, \frac{r+1}{2}\right]$ выполнено

$$
\left|h_{k, r}^{(q)}(x)\right| \leqslant \gamma(r, q)\left|\sin \frac{x}{2}\right|^{(k-q)_{+}}, \quad a_{+}=\max \{a, 0\}
$$

(при $k \leqslant q$ неравенство очевидно, а при $k>q$ нужно проинтегрировать $k-q$ раз по $[0, x]$ это неравенство при $k=q$ ), то из предыдущего рассуждения получаем следующее утверждение.

ЛЕмма 2. Для любого набора узлов $\Pi=\left\{x_{s}\right\}$ и чисел $\left\{y_{s, \nu}\right\}, 1 \leqslant s \leqslant m$, $0 \leqslant \nu \leqslant r$, существует единственный тригонометрический полином $\tau$ со спектром на $[p, n]$, где $n-p=m(r+1)-1$, удовлетворяющий условиям $\tau^{(\nu)}\left(x_{s}\right)=y_{s, \nu}, 1 \leqslant s \leqslant m, 0 \leqslant \nu \leqslant r$. При этом для всех $x \in \mathbb{R} u q \in \mathbb{Z}_{+}$

$$
\left|\tau^{(q)}(x)\right| \leqslant \gamma(r, q, \Pi) \sum_{\nu=0}^{r} \sum_{s=1}^{m}\left|y_{s, \nu}\right| .
$$

Подобное утверждение справедливо и в случае алгебраических полиномов. Уточним его в случае двух узлов $(m=2)$.

ЛЕмма 3. При любом $r \in \mathbb{Z}_{+}$и любом $k \in[0, r]$ алгебраический полином $p_{k, r}$ степени $2 r+1$, определяемьй условиями

$$
p_{k, r}^{(\nu)}=0, \quad \nu \neq k, \quad p_{k, r}^{(k)}(0)=1, \quad p_{k, r}^{(\nu)}(1)=0, \quad 0 \leqslant \nu \leqslant r,
$$

обладает следующими свойствами на $[0,1]$ :

$$
\frac{x^{k}}{k !}(1-x)^{r+1} \leqslant p_{k, r}(x) \leqslant \frac{x^{k}}{k !}\left(1-x^{r+1-k}\right), \quad\left|p_{k, r}^{(\nu)}(x)\right| \leqslant \gamma(r, \nu) x^{(k-\nu)_{+}} .
$$

ДокаЗАТЕЛЬСтво. При $r=0$ имеем $p_{0,0}(x)=1-x$. Считаем далее, что $r \in \mathbb{N}$.

При $k=0$ имеем $p_{0, r}(0)=1, p_{0, r}^{(\nu)}(0)=0,1 \leqslant \nu \leqslant r, p_{0, r}^{(\nu)}(1)=0,0 \leqslant \nu \leqslant r$. Проверим, что $p_{0, r}^{\prime}(x)<0$ на $(0,1)$.

Если бы полином $p_{0, r}^{\prime}$ степени $2 r$, уже имеющий $2 r$ нулей в концах отрезка $[0,1]$, имел бы еще один нуль, то $p_{0, r}=$ const, чего быть не может. Поэтому $p_{0, r}^{\prime}(x)<0$ и $0<p_{0, r}(x)<1$ на $(0,1)$.

Пусть теперь $k \in[1, r]$, при этом $p_{k, r}^{(k)}(0)=1$. Проверим, что $p_{k, r}(x) \leqslant \frac{x^{k}}{k !}$ на отрезке $[0,1]$.

Для этого представим полином в виде

$$
p_{k, r}(x)=\frac{x^{k}}{k !}+x^{r+1} \sum_{\nu=0}^{r} a_{\nu}(x-1)^{\nu}
$$

и вычислим коэффициенты $\left\{a_{\nu}\right\}: a_{0}=p_{k, r}(1)-\frac{1}{k !}=-\frac{1}{k !}$, а при $\nu \geqslant 1$

$$
\begin{aligned}
a_{\nu} & =\frac{1}{\nu !} \frac{d^{\nu}}{d x^{\nu}}\left\{\frac{p_{k, r}(x)-\frac{x^{k}}{k !}}{x^{r+1}}\right\}_{x=1}=\frac{-1}{\nu ! k !} \frac{d^{\nu}}{d x^{\nu}}\left\{x^{k-r-1}\right\}_{x=1} \\
& =\frac{(-1)^{\nu+1}}{\nu ! k !}(r-k+\nu) \cdots(r-k+1) .
\end{aligned}
$$


Следовательно, $a_{\nu}(x-1)^{\nu}<0$ при $\nu \in[0, r]$ и $x \in(0,1)$. Поэтому $p_{k, r}(x)<$ $\frac{x^{k}}{k !}+a_{0} x^{r+1}=\frac{x^{k}}{k !}\left(1-x^{r+1-k}\right)$.

Аналогично оцениваем $p_{k, r}$ снизу. Имеем

$$
\begin{gathered}
p_{k, r}(x)=(1-x)^{r+1} \sum_{\nu=k}^{r} b_{\nu} x^{\nu}, \\
b_{\nu}=\frac{1}{\nu !} \frac{d^{\nu}}{d x^{\nu}}\left\{p_{k, r}(x)(1-x)^{-r-1}\right\}_{x=0}=\frac{1}{k !(\nu-k) !} p_{k, r}^{(k)}(0) \frac{d^{\nu-k}}{d x^{\nu-k}}\left\{(1-x)^{-r-1}\right\}_{x=0} \\
=\frac{1}{k !(\nu-k) !}(r+1)(r+2) \cdots(r+\nu-k)>0 .
\end{gathered}
$$

Так что на интервале $(0,1)$ имеем

$$
p_{k, r}(x)>b_{k}(1-x)^{r+1} x^{k}=\frac{x^{k}}{k !}(1-x)^{r+1} .
$$

Осталось применить неравенство А. А. Маркова для производных алгебраических полиномов степени $n$ (см., например, [1], [2, п. 5.4.6]):

$$
\max _{0 \leqslant t \leqslant b}\left|p^{(\nu)}(t)\right| \leqslant\left(\frac{2}{b} n^{2}\right)^{\nu} \max _{0 \leqslant t \leqslant b}|p(t)| .
$$

В рассматриваемом случае $n=2 r+1$, а $b=x$ при $\nu \leqslant k$ и $b=1$ при $\nu>k$.

Вернемся к рассмотрению периодического случая.

Лемма 4. Существует абсолютная константа $c>1$ такая, что для любого $r \in \mathbb{Z}_{+}$, для любых наборов $\Pi=\left\{x_{s}\right\} u\left\{y_{s, \nu}\right\}, 1 \leqslant s \leqslant m, 0 \leqslant \nu \leqslant r$ можно построить такой тригонометрический полином высокого порядка, зависящего от $r$ и $\delta=\min _{s}\left|x_{s}-x_{s+1}\right|$, который удовлетворяет условиям

$$
\tau^{(\nu)}\left(x_{s}\right)=y_{s, \nu}, \quad 1 \leqslant s \leqslant m, \quad 0 \leqslant \nu \leqslant r, \quad\|\tau\| \leqslant c \max _{s, \nu}\left|y_{s, \nu}\right| .
$$

При $r=0$ число с можно считать сколь угодно близким $\kappa 1$, но тогда порядок полинома зависит еще от разности с-1.

ДокАЗАТЕЛЬСтво. По предположению имеем $x_{1}<x_{2}<\cdots<x_{m}<x_{m+1}=$ $x_{1}+2 \pi$. Считаем, что $y_{m+1, \nu}=y_{1, \nu}, 0 \leqslant \nu \leqslant r$. Построим $2 \pi$-периодический (эрмитов) сплайн $h$ степени $2 r+1$ из класса $C^{r}(\mathbb{T})$. Он определяется условиями $h^{(\nu)}\left(x_{s}\right)=y_{s, \nu}, 1 \leqslant s \leqslant m+1,0 \leqslant \nu \leqslant r$. На отрезке $\left[x_{s}, x_{s+1}\right]$ (где $x_{s+1}-x_{s}=\Delta x_{s}, p_{\nu, r}-$ полином из леммы 3) имеем

$$
h(x)=\sum_{\nu=0}^{r}\left\{y_{s, \nu} p_{\nu, r}\left(\frac{x-x_{s}}{\Delta x_{s}}\right)\left(\Delta x_{s}\right)^{\nu}+y_{s+1, \nu} p_{\nu, r}\left(\frac{x_{s+1}-x}{\Delta x_{s}}\right)\left(\Delta x_{s}\right)^{\nu}(-1)^{\nu}\right\},
$$

так как, например, при $q \leqslant r$

$$
h^{(q)}\left(x_{s+1}\right)=\sum_{\nu=0}^{r}\left\{y_{s, \nu} p_{\nu, r}^{(q)}(1)\left(\Delta x_{s}\right)^{\nu-q}+y_{s+1, \nu} p_{\nu, r}^{(q)}(0)\left(\Delta x_{s}\right)^{\nu-q}(-1)^{q+\nu}\right\}=y_{s+1, q} .
$$


В силу леммы 3 при $x \in\left[x_{s}, x_{s+1}\right]$ получаем

$$
\begin{aligned}
|h(x)| & \leqslant \sum_{\nu=0}^{r}\left\{\left|y_{s, \nu}\right| \frac{\left(x-x_{s}\right)^{\nu}}{\nu !}+\left|y_{s+1, \nu}\right| \frac{\left(x_{s+1}-x\right)^{\nu}}{\nu !}\right\} \\
& \leqslant \max _{\nu}\left\{\left|y_{s, \nu}\right|,\left|y_{s+1, \nu}\right|\right\} \sum_{\nu=0}^{r} \frac{1}{\nu !}\left\{\left(x-x_{s}\right)^{\nu}+\left(x_{s+1}-x\right)^{\nu}\right\} \\
& \leqslant \max _{\nu}\left\{\left|y_{s, \nu}\right|,\left|y_{s+1, \nu}\right|\right\} \sum_{\nu=0}^{r} \frac{1}{\nu !}\left(\Delta x_{s}\right)^{\nu} \leqslant e^{2 \pi} \max _{\nu}\left\{\left|y_{s, \nu}\right|,\left|y_{s+1, \nu}\right|\right\} .
\end{aligned}
$$

При $r=0$ вместо $e^{2 \pi}$ можно поставить 1 .

При $q \geqslant 1$ на отрезке $\left[x_{s}, x_{s+1}\right]$ в силу той же леммы 3 имеем

$$
\begin{gathered}
\left|h^{(q)}(x)\right| \leqslant \gamma(r, q) \sum_{\nu=0}^{r}\left\{\left|y_{s, \nu}\right|\left(\frac{x-x_{s}}{\Delta x_{s}}\right)^{(\nu-q)_{+}}\left(\Delta x_{s}\right)^{\nu-q}\right. \\
\left.\quad+\left|y_{s+1, \nu}\right|\left(\frac{x_{s+1}-x}{\Delta x_{s}}\right)^{(\nu-q)_{+}}\left(\Delta x_{s}\right)^{\nu-q}\right\} \\
\leqslant \gamma(r, q) \sum_{\nu=0}^{r}\left\{\left|y_{s, \nu}\right|+\left|y_{s+1, \nu}\right|\right\}\left(\Delta x_{s}\right)^{\nu-q} .
\end{gathered}
$$

Поэтому при $q \geqslant 1$ и $\Delta x_{s} \geqslant \delta>0$ получаем

$$
\left\|h^{(q)}\right\| \leqslant \gamma_{1}(r, q) \frac{1}{\delta^{q}} \max _{1 \leqslant s \leqslant m, 0 \leqslant \nu \leqslant r}\left|y_{s, \nu}\right| .
$$

По теореме Джексона для любого $n \in \mathbb{Z}_{+}$существует полином порядка не выше $n$ такой, что

$$
\left\|h^{(\nu)}-\tau_{n}^{(\nu)}\right\| \leqslant c \frac{\left\|h^{(r+1)}\right\|}{(n+1)^{r+1-\nu}}, \quad 0 \leqslant \nu \leqslant r+1 .
$$

Возьмем теперь эрмитов полином $\tau$, определяемый условиями

$$
\tau^{(\nu)}\left(x_{s}\right)=\left(h-\tau_{n}\right)^{(\nu)}\left(x_{s}\right), \quad 1 \leqslant s \leqslant m, \quad 0 \leqslant \nu \leqslant r .
$$

В силу леммы 3 и неравенства Джексона имеем

$$
\left\|\tau^{(q)}\right\| \leqslant \gamma(r, \Pi) \max _{s, \nu}\left|\left(h-\tau_{n}\right)^{(\nu)}\left(x_{s}\right)\right| \leqslant c \gamma(r, \Pi) \frac{\left\|h^{(r+1)}\right\|}{n+1} .
$$

Положим теперь $\tilde{\tau}=\tau_{n}+\tau$ (порядок этого полинома равен $\left.\max \{n, m(r+1)-1\}\right)$. Тогда при $1 \leqslant s \leqslant m$ и $0 \leqslant \nu \leqslant r$ имеем

$$
\tilde{\tau}^{(\nu)}\left(x_{s}\right)=\tau_{n}^{(\nu)}\left(x_{s}\right)+\left(h-\tau_{n}\right)^{(\nu)}\left(x_{s}\right)=h^{(\nu)}\left(x_{s}\right)=y_{s, \nu}
$$

При этом для $q \in[0, r]$ получаем

$$
\begin{aligned}
\left\|\tilde{\tau}^{(q)}\right\| & \leqslant\left\|\tau_{n}^{(q)}\right\|+\left\|\tau^{(q)}\right\| \leqslant\left\|h^{(q)}-\tau_{n}^{(q)}\right\|+\left\|h^{(q)}\right\|+\left\|\tau^{(q)}\right\| \\
& \leqslant c \frac{\left\|h^{(r+1)}\right\|}{(n+1)^{r+1-q}}+\left\|h^{(q)}\right\|+c \gamma(r, \Pi) \frac{\left\|h^{(r+1)}\right\|}{n+1} .
\end{aligned}
$$


Учитывая неравенство (3) при $q=r+1$, получаем

$$
\left\|\tilde{\tau}^{(q)}\right\| \leqslant \gamma_{1}(r, \Pi) \frac{1}{(n+1) \delta^{r+1}} \max _{s, \nu}\left|y_{s, \nu}\right|+\left\|h^{(q)}\right\| .
$$

При $q=0$, как это следует из $(2)$, имеем

$$
\|\tilde{\tau}\| \leqslant\left\{\sum_{\nu=0}^{r} \frac{1}{\nu !}\left(\max _{s} \Delta x_{s}\right)^{\nu}+\gamma_{1}(r, \Pi) \frac{1}{(n+1) \delta^{r+1}}\right\} \max _{s, \nu}\left|y_{s, \nu}\right|
$$

и при достаточно большом $n$ полином $\tilde{\tau}$ является искомым.

Лемма 5. Пусть $f \in C^{r}(\mathbb{T}), r \in \mathbb{Z}_{+}, u \tau_{f, r}-$ эрмитов интерполяционный тригонометрический полином, определяемыи условиями $\tau_{f, r}^{(\nu)}\left(x_{s}\right)=f^{(\nu)}\left(x_{s}\right)$, $1 \leqslant s \leqslant m, 0 \leqslant \nu \leqslant r$ (его порядок не больше $\left.\left[\frac{m(r+1)+1}{2}\right]\right)$. Тогда для $x \in \mathbb{R} u$ $\nu \in[0, r]$ имеем

$$
\left|f^{(\nu)}(x)-\tau_{f, r}^{(\nu)}(x)\right| \leqslant \gamma(r, \Pi) \min _{s}\left|\sin \frac{x-x_{s}}{2}\right|^{r-\nu} \omega\left(f^{(r)} ; \min _{s}\left|\sin \frac{x-x_{s}}{2}\right|\right),
$$

$a\left\|\tau_{f, r}^{(r+1)}\right\| \leqslant \gamma(r, \Pi) \omega\left(f^{(r)} ; \pi\right)$.

ДокАЗАтЕльство. Основное в лемме - это неравенство для $(r+1)$-й производной.

Сначала из него выведем неравенство о приближении. Пусть для определенности $x \in\left[\frac{x_{s}+x_{s+1}}{2}\right]$. Тогда

$$
f^{(r)}(x)-\tau_{f, r}^{(r)}(x)=\left[f^{(r)}(x)-f^{(r)}\left(x_{s}\right)\right]+\left[\tau_{f, r}^{(r)}\left(x_{s}\right)-\tau_{f, r}^{(r)}(x)\right]
$$

и в силу очевидного неравенства и монотонности $\omega$ имеем

$$
\left|f^{(r)}(x)-f^{(r)}\left(x_{s}\right)\right| \leqslant \omega\left(f^{(r)} ;\left|x-x_{s}\right|\right) \leqslant \omega\left(f^{(r)} ; \frac{2}{\pi}\left|\sin \left(x-x_{s}\right)\right|\right),
$$

при этом

$$
\left|\tau_{f, r}^{(r)}\left(x_{s}\right)-\tau_{f, r}^{(r)}(x)\right| \leqslant \int_{x_{s}}^{x}\left|\tau_{f, r}^{(r+1)}(t)\right| d t \leqslant \gamma(r, \Pi) \omega\left(f^{(r)} ; \pi\right)\left(x-x_{s}\right)
$$

и необходимо воспользоваться известным неравенством (см. ниже формулу (7) при $k=1$ )

$$
\omega(f ; c) h \leqslant 2 c \omega(f ; h) .
$$

Таким образом, искомое неравенство при $\nu=r$ доказано.

Далее можно либо интегрировать это неравенство $r$ раз по $\left[x_{s}, x\right]$, либо сразу воспользоваться формулой Тейлора для $f^{(\nu)}-\tau_{f, r}^{(\nu)}$ в окрестности точки $x_{s}$.

Неравенство для $(r+1)$-й производной полинома $\tau_{f, r}$ можно доказать индукцией по $m$ (вместо $\tau_{f, r}$ будем записывать $\left.\tau_{f, r, m}\right)$.

При $m=1$ нужно лишь учесть, что при $k \in[1, r]$ существует число $\xi_{k}$ такое, что $f^{(k)}\left(\xi_{k}\right)=0$. Тогда при $k \leqslant r-1$ имеем

$$
\begin{aligned}
\left\|f^{(k)}\right\| & =\max _{x}\left|\int_{\xi_{k}}^{x} f^{(k+1)}(t) d t\right| \leqslant \pi\left\|f^{(k+1)}\right\| \leqslant \pi^{r-k}\left\|f^{(r)}\right\| \\
& =\pi^{r-k}\left\|f^{(r)}(\cdot)-f^{(r)}\left(\xi_{r}\right)\right\| \leqslant \pi^{r-k} \omega\left(f^{(r)} ; \pi\right) .
\end{aligned}
$$


Норма производной тригонометрического полинома Тейлора при $r=2 n$ (см. лемму 1 ), а также и при $r=2 n-1$, когда $h_{0, r} \equiv 1, h_{k, r}=h_{k+1, r}^{\prime}$, следующая:

$$
\begin{aligned}
\left\|\sum_{k=0}^{r} f^{(k)}\left(x_{1}\right) h_{k, r}^{(r)}\left(\cdot-x_{1}\right)\right\| & =\left\|\sum_{k=1}^{r} f^{(k)}\left(x_{1}\right) h_{k, r}^{(r)}\left(\cdot-x_{1}\right)\right\| \leqslant \gamma(r) \sum_{k=1}^{r}\left\|f^{(k)}\right\| \\
& \leqslant \gamma_{1}(r)\left\|f^{(r)}\right\| \leqslant \gamma_{1}(r) \omega\left(f^{(r)} ; \pi\right) .
\end{aligned}
$$

Однако при $r=2 n$, например,

$$
\tau_{f, r, m}(x)=\tau_{f, r, m-1}+e^{-i\left[\frac{1}{2}(m-1)(r+1)\right] x} \prod_{s=1}^{m-1}\left(e^{i x}-e^{i x_{s}}\right)^{r+1} \sum_{k=0}^{2 n} a_{k} h_{k, 2 n}\left(x-x_{m}\right) .
$$

Теперь легко доказать такие же оценки для $\left|a_{k}\right|$, из которых и следует искомая оценка для $\left|\tau_{f, r, m}^{(r+1)}\right|$ (см. подобную лемму 5 в [3] для алгебраических полиномов).

Хорошо известна теорема Джексона-Стечкина: для любой функции $f \in$ $C(\mathbb{T})$ и любых $k \in \mathbb{Z}_{+}$и $n \in \mathbb{Z}_{+}$существует полином $\tau_{n}$ порядка не выше $n$, удовлетворяющий неравенству

$$
\left\|f-\tau_{n}\right\| \leqslant \gamma(k) \omega_{k}\left(f ; \frac{\pi}{n+1}\right)
$$

где $\omega_{0}(f)=\|f\|$, а при $k \geqslant 1$

$$
\omega_{k}(f ; h)=\sup _{0<\delta \leqslant h}\left\|\sum_{\nu=0}^{k} \frac{k !}{\nu !(k-\nu) !}(-1)^{\nu} f(\cdot+\nu \delta)\right\|
$$

- модуль гладкости функции $f$ (шага $h>0$ ).

Понадобятся следующие свойства $\omega_{k}$ (см., например, [1] или [2]):

$$
\begin{array}{cc}
\omega_{k+1}(f ; h) \leqslant 2 \omega_{k}(f ; h), & \omega_{k+1}(f ; h) \leqslant h \omega_{k}\left(f^{\prime} ; h\right), \\
\omega_{k}(f ; \lambda h) \leqslant(\lambda+1)^{k} \omega_{k}(f ; h), & \omega_{k}(f ; c) h^{k} \leqslant(2 c)^{k} \omega_{k}(f ; h), \\
\lambda>0, & 0<h \leqslant c .
\end{array}
$$

Лемма 6. Если для любой функции $f \in C(\mathbb{T})$ существует полином $\tau_{n}$ порядка не выше $n$ такой, что

$$
\left\|f-\tau_{n}\right\|=\varepsilon_{n}(f),
$$

то для любой функции $f \in C^{r}(\mathbb{T}), r \in \mathbb{Z}_{+}, u \nu \in[0, r]$ имеем

$$
\left\|f^{(\nu)}-\tau_{n}^{(\nu)}\right\| \leqslant \gamma(r)\left(\varepsilon_{n}\left(f^{(\nu)}\right)+n^{\nu} \varepsilon_{n}(f)\right)
$$

и для любого $s \in \mathbb{Z}_{+}$

$$
\left\|\tau_{n}^{(r+s)}\right\| \leqslant \gamma(r, s) n^{r+s}\left(\varepsilon_{n}(f)+\omega_{r+s}\left(f ; \frac{\pi}{n+1}\right)\right) .
$$


ДокАЗАТЕльСтво. В случае, когда $\varepsilon_{n}(f)=E_{n}^{\mathbb{T}}(f)$ и $\tau_{n}$ - полином наилучшего приближения, кроме того, $\varepsilon_{n}(f) \leqslant \frac{\pi}{2} \frac{1}{(n+1)^{\nu}} \varepsilon_{n}\left(f^{(\nu)}\right)$, эта лемма известна (см. [1] или [2]).

Рассмотрим полином Валле Пуссена $\tau_{1,2 n}(f)$ порядка $2 n$, для которого

$$
\left\|f-\tau_{1,2 n}(f)\right\| \leqslant 4 E_{n}^{\mathbb{T}}(f) \leqslant 4 \varepsilon_{n}(f) .
$$

Однако $\tau_{1,2 n}\left(f^{(\nu)}\right)=\tau_{1,2 n}^{(\nu)}(f)$ при $\nu \in[0, r]$, так как $\tau_{1,2 n}(f)-$ сверточный оператор. Тогда

$$
\left\|f^{(\nu)}-\tau_{1,2 n}^{(\nu)}(f)\right\| \leqslant 4 \varepsilon_{n}\left(f^{(\nu)}\right), \quad 0 \leqslant \nu \leqslant r .
$$

Кроме того,

$$
\left\|\tau_{n}(f)-\tau_{1,2 n}(f)\right\| \leqslant\left\|f-\tau_{n}(f)\right\|+\left\|f-\tau_{1,2 n}(f)\right\| \leqslant 5 \varepsilon_{n}(f),
$$

и в силу неравенства Бернштейна при любом $\nu \in \mathbb{Z}_{+}$имеем

$$
\left\|\tau_{n}^{(\nu)}(f)-\tau_{1,2 n}^{(\nu)}(f)\right\| \leqslant 5(2 n)^{\nu} \varepsilon_{n}(f) .
$$

Таким образом, при $\nu \in[0, r]$ получаем

$$
\left\|f^{(\nu)}-\tau_{n}^{(\nu)}(f)\right\| \leqslant\left\|f^{(\nu)}-\tau_{1,2 n}^{(\nu)}(f)\right\|+\left\|\tau_{1,2 n}^{(\nu)}(f)-\tau_{n}^{(\nu)}(f)\right\| \leqslant 4 \varepsilon_{n}\left(f^{(\nu)}\right)+5(2 n)^{\nu} \varepsilon_{n}(f) .
$$

Первое неравенство в лемме доказано.

В силу известного неравенства для полиномов (см. [1], [2, п. 8.2.4]) имеем

$$
\left\|\tau_{1,2 n}^{(r+s)}\right\| \leqslant \omega_{r+s}\left(\tau_{1,2 n} ; \frac{\pi}{2 n}\right) n^{r+s} .
$$

Однако

$$
\begin{aligned}
\omega_{r+s}\left(\tau_{1,2 n} ; h\right) & \leqslant \omega_{r+s}(f ; h)+\omega_{r+s}\left(f-\tau_{1,2 n} ; h\right) \leqslant \omega_{r+s}(f ; h)+2^{r+s}\left\|f-\tau_{1,2 n}\right\| \\
& \leqslant \omega_{r+s}(f ; h)+2^{r+s+2} E_{n}^{\mathbb{T}}(f) .
\end{aligned}
$$

Применяя неравенство (5) при $k=r+s$, получаем

$$
\left\|\tau_{1,2 n}^{(r+s)}\right\| \leqslant n^{r+s}\left[\omega_{r+s}\left(f ; \frac{\pi}{2 n}\right)+2^{r+s+2} \gamma(r+s) \omega_{r+s}\left(f ; \frac{\pi}{n+1}\right)\right] .
$$

Осталось воспользоваться неравенством (8) при $\nu=r+s$ :

$$
\left\|\tau_{n}^{(r+s)}\right\| \leqslant 5(2 n)^{r+s} \varepsilon_{n}(f)+\left\|\tau_{1,2 n}^{(r+s)}\right\| \leqslant \gamma_{1}(r, s) n^{r+s}\left(\varepsilon_{n}(f)+\omega_{r+s}\left(f ; \frac{\pi}{n+1}\right)\right) .
$$

Лемма 7. Для любых натуральных $k, m$ u $n$ существует алгебраический полином $p_{n}$ степени не выше $n$ такой, что для $x \in[-1,1]$ выполнено неравенство

$$
\left|x^{k}-x^{k+m} p_{n}(x)\right| \leqslant \gamma(k, m) \frac{1}{n^{k}} .
$$

ДокАЗАтЕЛьСтво см., например, в $[2$, п. 5.4.14, b)]. 
Лемма 8. Пусть $f \in C^{r}(\mathbb{T}) u f^{(\nu)}\left(x_{s}\right)=0,1 \leqslant s \leqslant m, 0 \leqslant \nu \leqslant r$. Тогда для любого $n \geqslant N(r, \Pi)$ и любого $k \in \mathbb{N}$ существует полином $\tau_{n}$ порядка не выше $n$ такой, что при $0 \leqslant \nu \leqslant r$

$$
\left\|f^{(\nu)}-\tau_{n}^{(\nu)}\right\| \leqslant \gamma(r, \Pi) \frac{1}{n^{r-\nu}} \omega_{k}\left(f^{(r)} ; \frac{\pi}{n}\right), \quad \tau_{n}^{(\nu)}\left(x_{s}\right)=0, \quad 1 \leqslant s \leqslant m .
$$

ДокАЗАТЕЛьство. В силу неравенства (5) найдется полином $\tau_{1, n}$ порядка не выше $n$ такой, что

$$
\left\|f-\tau_{1, n}\right\| \leqslant \gamma(k+r) \omega_{k+r}\left(f ; \frac{\pi}{n}\right) .
$$

В силу леммы 6 при $\nu \in[0, r]$ и свойств модуля гладкости (6) имеем

$$
\begin{aligned}
\left\|f^{(\nu)}-\tau_{1, n}^{(\nu)}\right\| & \leqslant \gamma_{1}(r, k)\left[\omega_{k+r}\left(f^{(\nu)} ; \frac{\pi}{n}\right)+n^{\nu} \omega_{k+r}\left(f ; \frac{\pi}{n}\right)\right] \\
& \leqslant \gamma_{1}(r, k)\left[2^{\nu} \omega_{k+r-\nu}\left(f^{(\nu)} ; \frac{\pi}{n}\right)+\pi^{\nu} \omega_{k+r-\nu}\left(f^{(\nu)} ; \frac{\pi}{n}\right)\right] \\
& \leqslant \gamma_{2}(r, k) \frac{1}{n^{r-\nu}} \omega_{k}\left(f^{(r)} ; \frac{\pi}{n}\right)
\end{aligned}
$$

Из условия леммы $f^{(\nu)}\left(x_{s}\right)=0$ теперь получаем

$$
\left|\tau_{1, n}^{(\nu)}\left(x_{s}\right)\right| \leqslant \gamma_{2}(r, k) \frac{1}{n^{r-\nu}} \omega_{k}\left(f^{(r)} ; \frac{\pi}{n}\right), \quad 1 \leqslant s \leqslant m, \quad 0 \leqslant \nu \leqslant r .
$$

Пусть $X_{1}(x)$ - тригонометрический полином с простыми нулями $\left\{x_{s}\right\}, 1 \leqslant$ $s \leqslant m$, наименьшего диаметра спектра. При четном $m$ имеем $X_{1}(x)=$ $\prod_{s=1}^{m} \sin \frac{x-x_{s}}{2}$, а при нечетном $m$ спектр полинома $X_{1}$ лежит в $\left[-\frac{m-1}{2}, \frac{m+1}{2}\right]$. Порядок спектра не более $\left[\frac{m+1}{2}\right]$ (см. начало настоящего параграфа).

Будем теперь делить $\tau_{1, n}$ на $X_{1}$ (см. свойство IV), если, конечно, $n \geqslant\left[\frac{m+1}{2}\right]$ :

$$
\tau_{1, n}(x)=a_{0}(x) X_{1}(x)+b_{0}(x),
$$

где $a_{0}$ - полином порядка не выше $n-\left[\frac{m+1}{2}\right]$, а $b_{0}$ однозначно определяется значениями $b_{0}\left(x_{s}\right)=\tau_{1, n}\left(x_{s}\right), 1 \leqslant s \leqslant m$. Если $n \geqslant 2\left[\frac{m+1}{2}\right]$, то и $a_{0}$ можно разделить на $X_{1}$. И так далее. Получим представление

$$
\tau_{1, n}(x)=\sum_{p=0}^{N_{1}} b_{p}(x) X_{1}^{p}(x) .
$$

В силу леммы 2 при $r=0$ и $q \in[0, r]$ (см., кроме того, (9)) имеем

$$
\left\|b_{0}^{(q)}\right\| \leqslant \gamma\left(r, X_{1}\right) \frac{1}{n^{r}} \omega_{k}\left(f^{(r)} ; \frac{\pi}{n}\right) .
$$

После дифференцирования тождества (11) и подстановки $x=x_{s}$ видно, что $b_{1}\left(x_{s}\right), 1 \leqslant s \leqslant m$, определяются значениями $\tau_{1, n}^{\prime}\left(x_{s}\right)$ и $b_{0}^{\prime}\left(x_{s}\right)$ при $1 \leqslant s \leqslant m$. В силу (10) при $\nu=1$ и леммы 2 имеем

$$
\left\|b_{1}^{(q)}\right\| \leqslant \gamma_{1}\left(r, k, X_{1}\right) \frac{1}{n^{r-1}} \omega_{k}\left(f^{(r)} ; \frac{\pi}{n}\right) .
$$


По индукции при $\nu$ и $q \in[0, r]$ получаем

$$
\left\|b_{\nu}^{(q)}\right\| \leqslant \gamma_{2}\left(r, k, X_{1}\right) \frac{1}{n^{r-\nu}} \omega_{k}\left(f^{(r)} ; \frac{\pi}{n}\right) .
$$

Считаем далее, что $\left\|X_{1}\right\|=1$. В силу леммы 7 имеем $\left\|X_{1}^{\nu}-X_{1}^{r+1} p_{n}\left(X_{1}\right)\right\|=$ $O\left(\frac{1}{n^{\nu}}\right)$. Следовательно, после умножения этого равенства на $b_{\nu}$ получаем

$$
\left\|b_{\nu} X_{1}^{\nu}-X_{1}^{r+1} b_{\nu} p_{n}\left(X_{1}\right)\right\|=O\left(\frac{1}{n^{r}} \omega_{k}\left(f^{(r)} ; \frac{\pi}{n}\right)\right) .
$$

Таким образом, каждое слагаемое в сумме (11) при $p \leqslant r$ заменяем тригонометрическим полиномом, который делится без остатка на $X_{1}^{r+1}$. Получим полином $\tau_{2, N}$, порядок $N$ которого принадлежат отрезку $\left[n,(2 n+r+3)\left[\frac{m+1}{2}\right]\right]$, при этом

$$
\left\|\tau_{1, n}-\tau_{2, N}\right\|=O\left(\frac{1}{n^{r}}\right) \omega_{k}\left(f^{(r)} ; \frac{\pi}{n}\right)=O\left(\frac{1}{N^{r}}\right) \omega_{k}\left(f^{(r)} ; \frac{\pi}{N}\right) .
$$

Последнее равенство легко доказать, используя первое неравенство в (7).

В силу неравенства Бернштейна при $\nu \in[0, r]$ имеем

$$
\left\|\tau_{1, n}^{(\nu)}-\tau_{2, N}^{(\nu)}\right\| \leqslant \gamma_{3}(r, k, \Pi) \frac{1}{N^{r-\nu}} \omega_{k}\left(f^{(r)} ; \frac{\pi}{N}\right) .
$$

Из неравенства (9) при $\nu \in[0, r]$ теперь получаем

$$
\left\|f^{(\nu)}-\tau_{2, N}^{(\nu)}\right\| \leqslant\left(\gamma_{2}(r, k)+\gamma_{3}(r, k, \Pi)\right) \frac{1}{N^{r-\nu}} \omega_{k}\left(f^{(r)} ; \frac{\pi}{n}\right) .
$$

Лемма 9. Пусть $\left\{\phi_{k}\right\}_{k=1}^{n}$ - линейно независимая система функиий из $C^{1}[a, b], a \Phi_{n}=\sum_{k=1}^{n} c_{k} \phi_{k}-$ полином по этой системе. Тогда при каждом $n$ и любом $x_{0} \in[a, b]$ имеем

$$
\sup _{f \in C^{\infty}[a, b]} \frac{1}{\|f\|} \inf _{\Phi_{n}:\left|\Phi_{n}^{\prime}\left(x_{0}\right)\right| \geqslant\left|f^{\prime}\left(x_{0}\right)\right|}\left\|f-\Phi_{n}\right\|=\infty .
$$

ДокАзАтельство. Предположим, что верхняя грань $\|f\|=\max _{x \in[a, b]}|f(x)|$ конечна. Тогда существуют число $c$ и полином $\Phi_{n}$ такие, что для всех $f \in$ $C^{\infty}[a, b]$

$$
\left\|\Phi_{n}\right\| \leqslant c\|f\|, \quad\left|\Phi_{n}^{\prime}\left(x_{0}\right)\right| \geqslant\left|f^{\prime}\left(x_{0}\right)\right| .
$$

В конечномерном пространстве все нормы эквивалентны, поэтому

$$
\left|\sum_{k=1}^{n} c_{k} \phi_{k}(a)\right|+\left\|\sum_{k=1}^{n} c_{k} \phi_{k}^{\prime}\right\| \leqslant \gamma(n)\left\|\sum_{k=1}^{n} c_{k} \phi_{k}\right\| .
$$

Отсюда для любой функции $f \in C^{\infty}[a, b]$ имеем

$$
\left|f^{\prime}\left(x_{0}\right)\right| \leqslant\left|\Phi_{n}^{\prime}\left(x_{0}\right)\right| \leqslant\left|\sum_{k=1}^{n} c_{k} \phi_{k}(a)\right|+\left\|\sum_{k=1}^{n} c_{k} \phi_{k}^{\prime}\right\| \leqslant \gamma(n)\left\|\sum_{k=1}^{n} c_{k} \phi_{k}\right\| \leqslant c \gamma(n)\|f\| .
$$

Однако это не выполняется уже для функции $f(x)=\sin \left(\lambda\left(x-x_{0}\right)\right)$ при $\lambda>$ $c \gamma(n)$. 
Теперь для доказательства теорем об односторонних аппроксимациях построим специальные полиномы. Далее функции и полиномы предполагаются вещественными.

Лемма 10. Существует абсолютная константа $c>1$ такая, что при любом $n \in \mathbb{N}$ можно построить нечетный тригонометрический полином $\tau_{n}$ порядка не выше $n$, удовлетворяющий на отрезке $[0, \pi]$ неравенству

$$
\min \{1, n \sin x\} \leqslant \tau_{n}(x) \leqslant c \min \{1, n \sin x\}
$$

и равенству $\tau_{n}(\pi-x)=\tau_{n}(x)$.

ДокАЗАТЕЛЬСтво. В качестве искомого полинома $\tau_{n}$ возьмем полином Джексона функции

$$
\Psi_{\varepsilon, n}(x)=\frac{n \sin x}{1+\varepsilon n|\sin x|}
$$

при достаточно малом $\varepsilon>0$ и $n>\frac{1}{\varepsilon}$. Точнее, этот полином нужно умножить на 2 (см. ниже формулу (15)). Необходимые свойства интеграла Джексона можно найти, например, в [1, п. 5 гл. II, п. 2 гл. IV].

Учитывая, что для функции $\psi_{\varepsilon}(t)=\frac{t}{1+\varepsilon t}=\frac{1}{\varepsilon}\left(1-\frac{1}{1+\varepsilon t}\right)$ при $t \geqslant 0$ выполнены неравенства

$$
\left|\psi_{\varepsilon}(t)\right| \leqslant \frac{1}{\varepsilon}, \quad\left|\psi_{\varepsilon}^{\prime}(t)\right| \leqslant 1, \quad\left|\psi_{\varepsilon}^{\prime \prime}(t)\right| \leqslant 2 \varepsilon,
$$

при $n \geqslant \frac{1}{\varepsilon}$ получаем

$$
\left\|\Psi_{\varepsilon, n}\right\| \leqslant \frac{1}{\varepsilon}, \quad\left\|\Psi_{\varepsilon, n}^{\prime}\right\| \leqslant n=\Psi_{\varepsilon, n}^{\prime}(0), \quad\left\|\Psi_{\varepsilon, n}^{\prime \prime}\right\| \leqslant n+2 \varepsilon n^{2} \leqslant 3 \varepsilon n^{2} .
$$

В силу положительности ядра Джексона $\left(\tau_{n}=\tau_{n}\left(\Psi_{\varepsilon, n}\right)\right)$ имеем

$$
\left\|\tau_{n}\right\| \leqslant \frac{1}{\varepsilon}, \quad\left\|\tau_{n}^{\prime}\left(\Psi_{\varepsilon, n}\right)\right\|=\left\|\tau_{n}\left(\Psi_{\varepsilon, n}^{\prime}\right)\right\| \leqslant n, \quad\left\|\tau_{n}^{\prime \prime}\right\| \leqslant 3 \varepsilon n^{2} .
$$

Так же, как и $\Psi_{\varepsilon, n}$, полином $\tau_{n}$ нечетный, а в силу теоремы Лагранжа о среднем имеем

$$
\begin{gathered}
\left|\tau_{n}(x)\right|=\left|\tau_{n}(x)-\tau_{n}(0)\right| \leqslant n|x| \leqslant \pi n\left|\sin \frac{x}{2}\right|, \\
\left|\tau_{n}(x)\right|=\left|\tau_{n}(x)-\tau_{n}(\pi)\right| \leqslant n|\pi-x| \leqslant \pi n\left|\cos \frac{x}{2}\right| .
\end{gathered}
$$

Следовательно, на отрезке $[-\pi, \pi]$ выполнено соотношение

$$
\left|\tau_{n}(x)\right| \leqslant \min \left\{\frac{1}{\varepsilon}, \pi n\left|\sin \frac{x}{2}\right|, \pi n\left|\cos \frac{x}{2}\right|\right\} \leqslant \min \left\{\frac{1}{\varepsilon}, \frac{\pi n}{\sqrt{2}}|\sin x|\right\} .
$$

В силу теоремы Джексона имеем

$$
\left\|\Psi_{\varepsilon, n}^{\prime}-\tau_{n}^{\prime}\right\| \leqslant \frac{c}{n}\left\|\Psi_{\varepsilon, n}^{\prime \prime}\right\| \leqslant 3 c \varepsilon n .
$$

Отсюда получаем

$$
\tau_{n}^{\prime}(0) \geqslant \Psi_{\varepsilon, n}^{\prime}(0)-3 c \varepsilon n=n(1-3 c \varepsilon),
$$


и в силу формулы Тейлора

$$
\tau_{n}(x) \geqslant \tau_{n}^{\prime}(0)-\frac{1}{2}\left\|\tau_{n}^{\prime \prime}\right\| x^{2} \geqslant n(1-3 c \varepsilon) x-\frac{1}{2} x^{2} 3 \varepsilon n^{2} .
$$

Пусть теперь $x \in\left[0, \frac{\pi}{2}\right]$ и $n \sin x \leqslant 1$. Тогда $x \leqslant \frac{\pi}{2} \sin x \leqslant \frac{\pi}{2 n}$ и выполнено соотношение

$$
\tau_{n}(x) \geqslant n x\left[1-3 c \varepsilon-\frac{3}{2} \varepsilon n x\right] \geqslant n x\left[1-\varepsilon\left(3 c+\frac{3 \pi}{4}\right)\right] .
$$

Если $x \in\left[0, \frac{\pi}{2}\right]$ и $n \sin x \geqslant 1$, то в силу монотонности $\Psi_{\varepsilon, n}$ (или $\psi_{\varepsilon}$ ) имеем

$$
\Psi_{\varepsilon, n}(x) \geqslant \frac{1}{1+\varepsilon}
$$

Поскольку, кроме того,

$$
\left\|\Psi_{\varepsilon, n}-\tau_{n}\left(\Psi_{\varepsilon, n}\right)\right\| \leqslant \frac{c}{n}\left\|\Psi_{\varepsilon, n}^{\prime}-\tau_{n}\left(\Psi_{\varepsilon, n}^{\prime}\right)\right\| \leqslant \frac{c^{2}}{n^{2}}\left\|\Psi_{\varepsilon, n}^{\prime \prime}\right\| \leqslant 3 c^{2} \varepsilon,
$$

то выполнены неравенства

$$
\tau_{n}(x) \geqslant \Psi_{\varepsilon, n}(x)-3 c^{2} \varepsilon \geqslant \frac{1}{1+\varepsilon}-3 c^{2} \varepsilon .
$$

Выбираем теперь $\varepsilon \in\left(0, \frac{1}{3}\right)$ так, чтобы

$$
\varepsilon\left(3 c+\frac{3 \pi}{4}\right) \leqslant \frac{1}{2}, \quad 3 c^{2} \varepsilon \leqslant \frac{1}{1+\varepsilon}-\frac{1}{2} .
$$

Тогда при $n \sin x \leqslant 1$ (см. (13)) имеем $\tau_{n}(x) \geqslant \frac{1}{2} \sin x$, а при $n \sin x \geqslant 1$ (см. (14)) имеем $\tau_{n}(x) \geqslant \frac{1}{2}$, в результате на отрезке $\left[0, \frac{\pi}{2}\right]$ получаем

$$
\tau_{n}(x) \geqslant \frac{1}{2} \min \{1, n \sin x\}
$$

Поскольку $\Psi_{\varepsilon, n}(\pi-x)=\Psi_{\varepsilon, n}(x)$, то $\tau_{n}(\pi-x)=\tau_{n}(x)$ (оператор Джексона, как и всякий сверточный оператор, не расширяет спектр). При $n \geqslant \frac{1}{\varepsilon}$ и $x \in[0, \pi]$ получаем (см. (12))

$$
\frac{1}{2} \min \{1, n \sin x\} \leqslant \tau_{n}(x) \leqslant \frac{1}{\varepsilon} \min \{1, n \sin x\} .
$$

При $1 \leqslant n<\frac{1}{\varepsilon}$ берем $\tau_{1}(x)=n \sin x$. Тогда на отрезке $[0, \pi]$ имеем

$$
\min \{1, n \sin x\} \leqslant \tau_{1}(x) \leqslant \min \left\{\frac{1}{\varepsilon}, n \sin x\right\} \leqslant \frac{1}{\varepsilon} \min \{1, n \sin x\} .
$$

Рассмотрим теперь кусочно одностороннюю аппроксимацию. В следующей лемме дан ответ на вопрос о возможности такой аппроксимации. 
Лемма 11. Справедливы следующие утверждения:

а) если $f$ - ограниченная $2 \pi$-периодическая функиия, $x_{1}<x_{2}<\cdots<x_{m}<$ $x_{m+1}=x_{1}+2 \pi, m$ - четное число и функция $f$ непрерывна в точках $x_{s}$, $1 \leqslant s \leqslant m$, то для того, чтобы существовал полином $\tau_{f}$ с условиями

$$
\tau_{f}\left(x_{s}\right)=f\left(x_{s}\right), \quad(-1)^{s}\left(f(x)-\tau_{f}(x)\right)>0, \quad x \in\left(x_{s}, x_{s+1}\right), \quad 1 \leqslant s \leqslant m,
$$

необходимо и достаточно, чтобы при любом $s \in[1, m]$

$$
\varliminf_{x \rightarrow x_{s}}(-1)^{s} \frac{f(x)-f\left(x_{s}\right)}{x-x_{s}}>-\infty ;
$$

b) если $f \in C^{1}(\mathbb{T})$, то существует полином $\tau$ порядка не выше $\frac{m}{2}$ такой, что на интервалах $\left(x_{s}, x_{s+1}\right), 1 \leqslant s \leqslant m$, выполнено

$$
(-1)^{s}(f(x)-\tau(x))>0,
$$

$\omega\left(f^{\prime} ; \pi\right) \min _{\nu}\left|\sin \frac{x-x_{\nu}}{2}\right| \leqslant(-1)^{s}(f(x)-\tau(x)) \leqslant \gamma(\Pi) \omega\left(f^{\prime} ; \pi\right) \min _{\nu}\left|\sin \frac{x-x_{\nu}}{2}\right|$.

ДоказАТЕЛьство. а) Докажем необходимость. Имеем $\tau\left(x_{s}\right)=f\left(x_{s}\right), 1 \leqslant$ $s \leqslant m$. Пусть $x \in\left(x_{s}, x_{s+1}\right)$. Тогда при данном полиноме $\tau$ выполнено

$$
\begin{aligned}
(-1)^{s} \frac{f(x)-f\left(x_{s}\right)}{x-x_{s}} & =(-1)^{s} \frac{f(x)-\tau(x)}{x-x_{s}}+(-1)^{s} \frac{\tau(x)-\tau\left(x_{s}\right)}{x-x_{s}} \\
& >(-1)^{s} \frac{\tau(x)-\tau\left(x_{s}\right)}{x-x_{s}},
\end{aligned}
$$

откуда получаем

$$
\varliminf_{x \rightarrow x_{s}+0}(-1)^{s} \frac{f(x)-f\left(x_{s}\right)}{x-x_{s}} \geqslant(-1)^{s} \tau^{\prime}\left(x_{s}\right) .
$$

Аналогично при $x \in\left(x_{s-1}, x_{s}\right)$ и $s \in[2, m+1]$ имеем

$$
\begin{aligned}
(-1)^{s} \frac{f(x)-f\left(x_{s}\right)}{x-x_{s}} & =(-1)^{s-1} \frac{f(x)-\tau(x)}{x_{s}-x}+(-1)^{s} \frac{\tau(x)-\tau\left(x_{s}\right)}{x-x_{s}} \\
& >(-1)^{s} \frac{\tau(x)-\tau\left(x_{s}\right)}{x-x_{s}}
\end{aligned}
$$

и нужно лишь устремить $x$ к $x_{s}-0$.

Докажем достаточность указанного в лемме условия в каждой точке $x_{s}$. Пусть $x \in\left(x_{s}, x_{s+1}\right)$. Тогда $\sin \frac{x-x_{\nu}}{2}>0$ при $s \geqslant \nu \geqslant 1$ и $\sin \frac{x-x_{\nu}}{2}<0$ при $s<\nu \leqslant m$. Следовательно, если $h_{0}(x)=\prod_{\nu=1}^{m} \sin \frac{x-x_{\nu}}{2}$ (полином порядка $\frac{m}{2}$ ), то на $\left(x_{s}, x_{s+1}\right)$ имеем $(-1)^{s} h_{0}(x)>0$. Нужно доказать, что при $x \in \mathbb{R}$ и $x \neq x_{s}$, $1 \leqslant s \leqslant m$, для некоторого полинома $\tau$ порядка $\frac{m}{2}$ выполнено

$$
\frac{f(x)-\tau(x)}{h_{0}(x)}>0 .
$$

Пусть $\tau_{f}$ - полином, определяемый условиями $\tau_{f}\left(x_{s}\right)=f\left(x_{s}\right), 1 \leqslant s \leqslant m$ (его порядок не выше $\left.\frac{m}{2}\right)$. Проверим, что существует такое число $M$, что для всех $x \in \mathbb{R}$ и $x \neq x_{s}, 1 \leqslant s \leqslant m$, выполнено

$$
g(x)=\frac{f(x)-\tau_{f}(x)}{h_{0}(x)}>M .
$$


Имеем

$$
\begin{aligned}
\underline{\lim }_{x \rightarrow x_{s}} \frac{f(x)-\tau_{f}(x)}{h_{0}(x)} & =\underline{\lim }_{x \rightarrow x_{s}}\left(\frac{f(x)-f\left(x_{s}\right)}{h_{0}(x)}+\frac{\tau_{f}\left(x_{s}\right)-\tau_{f}(x)}{h_{0}(x)}\right) \\
& =\underline{\lim }_{x \rightarrow x_{s}} 2 \frac{f(x)-f\left(x_{s}\right)}{x-x_{s}} \frac{\sin \frac{x-x_{s}}{2}}{h_{0}(x)}-\frac{2 \tau_{f}^{\prime}\left(x_{s}\right)}{\prod_{\nu \neq s} \sin \frac{x_{s}-x_{\nu}}{2}} .
\end{aligned}
$$

Однако при любом $s \in[1, m]$ имеем

$$
(-1)^{s} h_{0}^{\prime}\left(x_{s}\right)=\frac{1}{2}(-1)^{s} \prod_{\nu \neq s} \sin \frac{x_{s}-x_{\nu}}{2}>0,
$$

и в силу условия леммы этот предел больше $-\infty$. Таким образом, функция $g$ ограничена снизу в достаточно малых окрестностях точек $\left\{x_{s}\right\}$, а значит, и всюду, так как числитель ограничен, а знаменатель $h_{0}-$ непрерывная функция и $h_{0}(x) \neq 0$ при $x \neq x_{s}$. Однако тогда при $\tau(x)=\tau_{f}(x)+M h_{0}(x)$ и $x \neq x_{s}$

$$
\frac{f(x)-\tau(x)}{h_{0}(x)}=g(x)-M>0 .
$$

b) Рассмотрим тот же полином $\tau(x)=\tau_{f}(x)+M h_{0}(x)$. Не уменьшая общности, считаем, что $f\left(x_{1}\right)=0$. Тогда (см. (4))

$$
\|f\| \leqslant \pi\left\|f^{\prime}\right\| \leqslant \omega\left(f^{\prime} ; \pi\right) .
$$

Как видно из доказательства достаточности в п. а), можно считать для $f \in$ $C^{1}(\mathbb{T})$, что

$$
|M| \leqslant \gamma_{1}(\Pi)\left(\left\|f^{\prime}\right\|+\left\|\tau_{f}^{\prime}\right\|\right) .
$$

Из леммы 2 при $r=0$ и $q=1$ следует, что

$\left\|\tau_{f}^{\prime}\right\| \leqslant \gamma(\Pi) \max _{s}\left|f\left(x_{s}\right)\right|=\gamma(\Pi) \max _{s}\left|f\left(x_{s}\right)-f\left(x_{1}\right)\right| \leqslant \pi \gamma(\Pi)\left\|f^{\prime}\right\| \leqslant \pi \gamma(\Pi) \omega\left(f^{\prime} ; \pi\right)$.

Поэтому при любом $x \in\left[x_{1}, x_{m+1}\right]$ и любом $s \in[1, m+1]$ имеем

$$
\begin{aligned}
|f(x)-\tau(x)| & =\left|\left[f(x)-f\left(x_{s}\right)\right]+\left[\tau_{f}\left(x_{s}\right)-\tau_{f}(x)\right]-M h_{0}(x)\right| \\
& \leqslant\left\|f^{\prime}\right\|\left|x-x_{s}\right|+\left\|\tau_{f}^{\prime}\right\|\left|x-x_{s}\right|+|M| \min _{\nu}\left|\sin \frac{x-x_{\nu}}{2}\right| .
\end{aligned}
$$

Таким образом, для всех $x \in \mathbb{R}$ получаем

$$
|f(x)-\tau(x)| \leqslant \gamma_{2}(\Pi) \omega\left(f^{\prime} ; \pi\right) \min _{\nu}\left|\sin \frac{x-x_{\nu}}{2}\right|,
$$

значит, при любом $\gamma>0$ и $x \in\left(x_{s}, x_{s+1}\right)$

$$
(-1)^{s}\left(f(x)-\tau(x)+\gamma h_{0}(x)\right) \geqslant \gamma \min _{\nu}\left|\sin \frac{x-x_{\nu}}{2}\right|-\gamma_{2}(\Pi) \omega\left(f^{\prime} ; \pi\right) \min _{\nu}\left|\sin \frac{x-x_{\nu}}{2}\right| .
$$

Выбирая $\gamma=\left(\gamma_{2}(\Pi)+1\right) \omega\left(f^{\prime} ; \pi\right)$, при $x \in\left(x_{s}, x_{s+1}\right)$ получаем

$$
\begin{gathered}
\omega\left(f^{\prime} ; \pi\right) \min _{\nu}\left|\sin \frac{x-x_{\nu}}{2}\right| \leqslant(-1)^{s}\left(f(x)-\tau(x)+\gamma h_{0}(x)\right) \\
\leqslant \gamma_{3}(\Pi) \omega\left(f^{\prime} ; \pi\right) \min _{\nu}\left|\sin \frac{x-x_{\nu}}{2}\right|
\end{gathered}
$$


Как видно из следующей леммы (см. утверждение а)), в последнем неравенстве вместо $\omega\left(f^{\prime} ; \pi\right)$ можно поставить $\omega_{k}\left(f^{\prime} ; \pi\right)$ при любом $k \geqslant 2$.

ЛЕмма 12. Справедливы следующие утверждения:

а) существует абсолютная константа с >0 такая, что для любой функиии $f \in C(\mathbb{T})$ и любого $k \in \mathbb{N}$ имеем

$$
\frac{1}{4} \omega(f ; \pi) \leqslant E_{0}^{\mathbb{T}}(f)=\min _{\lambda \in \mathbb{C}}\|f-\lambda\| \leqslant c \omega_{k}(f ; \pi) ;
$$

b) $п р и$ p $\leqslant$ u $h \in(0, \pi]$ имеем

$$
\omega_{p}\left(f ; h^{k}\right) \leqslant \gamma(p, k) \omega_{k}\left(f ; h^{p}\right) .
$$

ДокАзАтЕльство. а) Если $f=f_{1}+i f_{2}$, где $f_{1}$ и $f_{2}$ - непрерывные периодические и вещественные функции, то

$$
\omega(f ; \pi) \leqslant \omega\left(f_{1} ; \pi\right)+\omega\left(f_{2} ; \pi\right)=2 E_{0}^{\mathbb{T}}\left(f_{1}\right)+2 E_{0}^{\mathbb{T}}\left(f_{2}\right) \leqslant 4 E_{0}^{\mathbb{T}}(f),
$$

так как если $f_{1}\left(x_{1}\right)=\max f_{1}(x)$, а $f_{1}\left(x_{2}\right)=\min f_{1}(x)$ на периоде, то, считая, что $\left|x_{1}-x_{2}\right| \leqslant \pi$, имеем

$$
E_{0}^{\mathbb{T}}\left(f_{1}\right)=\frac{f_{1}\left(x_{1}\right)-f_{1}\left(x_{2}\right)}{2}=\frac{1}{2} \omega\left(f_{1} ; \pi\right) .
$$

Пусть для определенности $x_{1}<x_{2}$. Тогда на отрезке $\left[x_{1}, x_{1}+k \pi\right]$ функция $f_{1}(x)-\frac{1}{2}\left(f_{1}\left(x_{1}\right)+f_{1}\left(x_{2}\right)\right)$ имеет $k+1$ точек альтернанса. По теореме П. Л. Чебышева для алгебраических полиномов (см. [1] или [2]) получаем

$$
E_{0}^{\mathbb{T}}\left(f_{1}\right)=E_{k-1}\left(f_{1} ;\left[x_{1}, x_{1}+k \pi\right]\right) .
$$

По теореме Уитни-Сендова (см. [12], $c \leqslant 6$ ) имеем

$$
E_{k-1}\left(f_{1} ;\left[x_{1}, x_{1}+k \pi\right]\right) \leqslant c \omega_{k}\left(f_{1} ; \frac{k \pi}{k+1}\right) \leqslant c \omega_{k}\left(f_{1} ; \pi\right)
$$

Аналогичные неравенства справедливы для $f_{2}$ и $f$.

b) Ранее автором доказано следующее неравенство для любых функций на отрезке $[0, \pi]$ (см. $\left[2\right.$, п. 4.6.5c]): при $0<h \leqslant \min \left\{1,\left(\frac{\pi}{k}\right)^{\frac{1}{p}},\left(\frac{\pi}{p}\right)^{\frac{1}{k}}\right\}$

$$
\omega_{k}\left(f ; \frac{\pi}{k}\right) \omega_{p}\left(f ; h^{k}\right) \leqslant \gamma_{1}(p, k) \omega_{p}\left(f ; \frac{\pi}{p}\right)(\pi+1)^{k-p} \omega_{k}\left(f ; h^{p}\right) .
$$

Необходимо убрать зависимость постоянных множителей от функции $f$, т. е. доказать неравенство $\omega_{p}\left(f ; \frac{\pi}{p}\right) \leqslant \gamma_{2}(p, k) \omega_{k}\left(f ; \frac{\pi}{k}\right)$. Здесь важно, что функции периодические.

Воспользуемся свойствами модулей гладкости (6), (7) и утверждением а): $\omega_{p}\left(f ; \frac{\pi}{p}\right) \leqslant \omega_{p}(f ; \pi) \leqslant 2^{p-1} \omega(f ; \pi) \leqslant 4 c \cdot 2^{p-1} \omega_{k}(f ; \pi) \leqslant 4 c \cdot 2^{p-1}(k+1)^{k} \omega_{k}\left(f ; \frac{\pi}{k}\right)$. 


\section{§ 3. Доказательство теорем 1-4}

ДокАЗАТЕЛЬСтво теоремы 1. Утверждение b) является частным случаем $(m=1)$ леммы 5 .

Для доказательства утверждения а) используем факторизацию дифференциальных операторов по Фробениусу и обобщенную теорему Ролля, доказанную Пойа (см. [13, гл. IV], [14, разд. V, гл. 1]).

Лемма А. Пусть при $\lambda>0$ u $n \geqslant 2$ функиия $f \in C^{n}\left[0, \frac{\pi}{\lambda}\right)$ имеет $m \geqslant 3$ нулей на полуинтервале $\left[0, \frac{\pi}{\lambda}\right)$ с учетом кратностей. Тогда функиия $f^{\prime \prime}+\lambda^{2} f$ имеет на том же полуинтервале не менее $m-2$ нулей.

ДокАЗАТЕЛЬСтво. Не уменьшая общности, считаем $\lambda=1$ (преобразование подобия). Легко видеть, что

$$
f^{\prime \prime}(x)+f(x)=\frac{1}{\sin x} \frac{d}{d x}\left\{\sin ^{2} x \frac{d}{d x}\left(\frac{f(x)}{\sin x}\right)\right\} .
$$

Если $f(x) \neq 0$ на $(0, \pi)$ и, значит, $f^{(\nu)}(0)=0$ при $0 \leqslant \nu \leqslant m-1$, то по известной лемме Адамара частное $\frac{f(x)}{\sin x} \in C^{n-1}[0, \pi)$ имеет в нуле нуль кратности $m-1$, но тогда функция, записанная в фигурных скобках, имеет в нуле нуль кратности $m$. Следовательно, функция $f^{\prime \prime}+f$ имеет в нуле $m-2$ нулей (не менее).

Если функция $f$ имеет нули на интервале $(0, \pi)$, то используем аналогичное рассуждение с применением теоремы Ролля. Лемма доказана.

Как видно из доказательства, если нули $f$ лежат на $[0, \delta \pi]$ при $\delta<1$, то и нули $f^{\prime \prime}+f$ лежат на $[0, \delta \pi]$.

Положим

$$
\Delta_{n}(x)=f(x)-\sum_{k=0}^{2 n} f^{(k)}(0) h_{k, 2 n}(x)
$$

а при $x \in(0,2 \pi)$ пусть

$$
H(x)=\frac{\Delta_{n}(x)}{h_{2 n, 2 n}(x)}, \quad F(y)=\Delta_{n}(y)-H(x) h_{2 n, 2 n}(y) .
$$

Очевидно, что $\Delta_{n}^{(\nu)}(0)=0$ при $0 \leqslant \nu \leqslant 2 n, F^{(\nu)}(0)=0$ при $0 \leqslant \nu \leqslant 2 n-1$ и $F(x)=0$.

Если, кроме того, $F^{(2 n)}(0)=0$, то $\Delta_{n}^{(2 n)}(0)-H(x) h_{2 n, 2 n}^{(2 n)}(0)=0$ и $H(x)=0$, т. e.

$$
\Delta_{n}(x)=f(x)-\sum_{k=0}^{2 n} f^{(k)}(0) h_{k, 2 n}(x)=0
$$

и доказательство очевидно $(\theta=1)$. 
Считаем далее, что $F^{(2 n)}(0) \neq 0$ и $x \in\left(0, \frac{\pi}{n}\right)$. К функции $F$, имеющей $2 n+1$ нулей на отрезке $[0, x]$, применяем лемму А при $\lambda=n$. Тогда функция $F^{\prime \prime}+n^{2} F$ имеет на $\left[0, \frac{\pi}{n}\right)$ не менее $2 n-1$ нулей. При этом в нуле ровно $2 n-2$ нулей, так каK

$$
\left(F^{\prime \prime}+n^{2} F\right)_{x=0}^{(2 n-2)}=F^{(2 n)}(0) \neq 0,
$$

значит, хотя бы один нуль лежит в интервале $(0, x)$.

Если $n \geqslant 2$, то применяем лемму А при $\lambda=n-1$ к функции $F^{\prime \prime}+n^{2} F$. Продолжая таким же образом, получим, что существует число $\xi \in(0, x)$ такое, что $D_{2 n} F(\xi)=0$. Следовательно,

$$
\Delta_{n}(x)=H(x) h_{2 n, 2 n}(x)=\frac{D_{2 n} \Delta_{n}(\xi)}{D_{2 n} h_{2 n, 2 n}(\xi)} h_{2 n, 2 n}(x) .
$$

Очевидно, что для любого полинома $\tau$ порядка не выше $n$

$$
D_{2 n} \tau(x) \equiv(n !)^{2} \hat{\tau}(0)=(n !)^{2} \frac{1}{2 \pi} \int_{-\pi}^{\pi} \tau(t) d t .
$$

Для любой функции $f \in C^{2 n}$ выполнено

$$
D_{2 n} f(x)=q_{n} \frac{d^{2}}{d x^{2}} f(x)=\sum_{s=0}^{n} a_{s, n} f^{(2 s)}(x),
$$

а так как (см. лемму 1)

$$
h_{k, 2 n}(x)=\sum_{s=\left[\frac{k+1}{2}\right]}^{n} a_{s, n} h_{2 n, 2 n}^{(2 s-k)}(x),
$$

то при четном $k$ получаем

$$
\hat{h}_{k, 2 n}(0)=\sum_{s=\left[\frac{k+1}{2}\right]}^{n} a_{s, n} \frac{1}{2 \pi} \int_{-\pi}^{\pi} h_{2 n, 2 n}^{(2 s-k)}(x)=a_{\frac{k}{2}, n} \hat{h}_{2 n, 2 n}(0)=\frac{1}{(n !)^{2}} a_{\frac{k}{2}, n},
$$

а при нечетном $k$ имеем $\hat{h}_{k, 2 n}(0)=0$. При этом $D_{2 n} h_{2 n, 2 n} \equiv a_{n, n}=1$. Поэтому

$$
\begin{aligned}
D_{2 n} \Delta_{n}(\xi) & =D_{2 n} f(\xi)-\sum_{k=0}^{2 n} f^{(k)}(0) D_{2 n} h_{k, 2 n}(\xi) \\
& =D_{2 n} f(\xi)-\sum_{k=0}^{2 n} f^{(k)}(0)(n !)^{2} \hat{h}_{k, 2 n}(0) \\
& =D_{2 n} f(\xi)-\sum_{s=0}^{n} a_{s, n} f^{(2 s)}(0)=D_{2 n} f(\xi)-D_{2 n} f(0) .
\end{aligned}
$$

Таким образом, для любой функции $f \in C^{2 n}(\mathbb{T})$ и $x \in\left(0, \frac{\pi}{n}\right)$ существует $\xi \in$ $(0, x)$ такое, что

$$
f(x)-\sum_{k=0}^{2 n} f^{(k)}(0) h_{k, 2 n}(x)=\left(D_{2 n} f(\xi)-D_{2 n} f(0)\right) h_{2 n, 2 n}(x) .
$$

Подобное равенство имеет место, конечно, и при $x \in\left(-\frac{\pi}{n}, 0\right)$. 
ДОКАЗАТЕЛЬСТВО ТЕОРЕмЫ 2. Пусть $\tau_{f}$ - эрмитов полином, определяемый условиями $\tau_{f}^{(\nu)}\left(x_{s}\right)=f^{(\nu)}\left(x_{s}\right), 1 \leqslant s \leqslant m, 0 \leqslant \nu \leqslant r$ (его порядок не выше $\left.\left[\frac{m(r+1)}{2}\right]\right)$. В силу леммы 8 при $n \geqslant N(r, \Pi)$ найдется полином $\tau_{n}$ порядка не выше $n$ такой, что $\tau_{n}^{(\nu)}\left(x_{s}\right)=0,1 \leqslant s \leqslant m, 0 \leqslant \nu \leqslant r$ и

$$
\left\|f^{(\nu)}-\tau_{f}^{(\nu)}-\tau_{n}^{(\nu)}\right\| \leqslant \gamma(r, k, \Pi) \frac{1}{n^{r-\nu}} \omega_{k}\left(\left(f-\tau_{f}\right)^{(r)} ; \frac{1}{n}\right) .
$$

Однако в силу свойств модулей гладкости и неравенства Бернштейна имеем

$$
\omega_{k}\left(\tau_{f}^{(r)} ; h\right) \leqslant\left\|\tau_{f}^{(r+k)}\right\| h^{k} \leqslant\left(\frac{m(r+1)}{2}\right)^{k-1}\left\|\tau_{f}^{(r+1)}\right\| h^{k},
$$

а после применения леммы 5 и леммы 12, b) (см., кроме того, (7)) получаем

$$
\begin{aligned}
\left\|\tau_{f}^{(r+1)}\right\| h^{k} & \leqslant \gamma_{1}(r, k, \Pi) h^{k} \omega\left(f^{(r)} ; \pi\right) \leqslant \gamma_{2}(r, k, \Pi) h^{k} \omega_{k}\left(f^{(r)} ; \pi\right) \\
& \leqslant(2 \pi)^{k} \gamma_{2}(r, k, \Pi) \omega_{k}\left(f^{(r)} ; h\right) .
\end{aligned}
$$

Таким образом, при $0<h \leqslant \pi$ имеем

$$
\omega_{k}\left(\left(f-\tau_{f}\right)^{(r)} ; h\right) \leqslant \omega_{k}\left(f^{(r)} ; h\right)+\omega_{k}\left(\tau_{f}^{(r)} ; h\right) \leqslant \gamma_{3}(r, k, \Pi) \omega_{k}\left(f^{(r)} ; h\right),
$$

а значит, при $0 \leqslant \nu \leqslant r$

$$
\left\|f^{(\nu)}-\tau_{f}^{(\nu)}-\tau_{n}^{(\nu)}\right\| \leqslant \gamma_{4}(r, k, \Pi) \frac{1}{n^{r-\nu}} \omega_{k}\left(f^{(r)} ; \frac{1}{n}\right) .
$$

Пусть $\tau_{1, n}=\tau_{n}+\tau_{f}$. Тогда $\tau_{1, n}^{(\nu)}\left(x_{s}\right)=f^{(\nu)}\left(x_{s}\right), 1 \leqslant s \leqslant m, 0 \leqslant \nu \leqslant r$, и

$$
\left\|f^{(\nu)}-\tau_{1, n}^{(\nu)}\right\| \leqslant \gamma_{4}(r, k, \Pi) \frac{1}{n^{r-\nu}} \omega_{k}\left(f^{(r)} ; \frac{1}{n}\right),
$$

а если учесть лемму 6 при $s=1$, то получим

$$
\left\|\tau_{1, n}^{(r+1)}\right\| \leqslant \gamma_{5}(r, k, \Pi) n \omega_{k}\left(f^{(r)} ; \frac{1}{n}\right) .
$$

При любом $x \in\left[x_{1}, x_{m+1}\right]$ в силу формулы Тейлора и неравенства (16) при $\nu=r$ и любом $s \in[1, m+1]$ имеем

$$
\begin{aligned}
\mid f^{(\nu)}(x)- & \tau_{1, n}^{(\nu)}(x)\left|\leqslant \frac{1}{(r-\nu) !}\left\|f^{(r)}-\tau_{1, n}^{(r)}\right\|\right| x-\left.x_{s}\right|^{r-\nu} \\
& \leqslant \gamma_{4} \frac{1}{(r-\nu) !}\left(\frac{\pi}{2}\right)^{r-\nu}\left|\sin \frac{x-x_{s}}{2}\right|^{r-\nu} \omega_{k}\left(f^{(r)} ; \frac{1}{n}\right),
\end{aligned}
$$

а значит, при $0 \leqslant \nu \leqslant r$ для всех $x \in \mathbb{R}$ получаем

$$
\begin{aligned}
& \left|f^{(\nu)}(x)-\tau_{1, n}^{(\nu)}(x)\right| \leqslant \gamma_{6} \min _{s}\left\{\frac{1}{n},\left|\sin \frac{x-x_{s}}{2}\right|\right\}^{r-\nu} \omega_{k}\left(f^{(r)} ; \frac{1}{n}\right), \\
& \left|f^{(\nu)}(x)-\tau_{1, n}^{(\nu)}(x)\right| \leqslant 2^{k} \gamma_{6} \min _{s}\left\{\frac{1}{n},\left|\sin \frac{x-x_{s}}{2}\right|\right\}^{r-\nu}\left\|f^{(r)}\right\| .
\end{aligned}
$$


Пусть теперь $f \in C^{r+k}(\mathbb{T})$. Получим сначала два результата частного характера, которые затем проинтерполируем.

Используя неравенство (17), получаем

$$
\begin{aligned}
\mid f^{(r)}(x)- & \tau_{1, n}^{(r)}(x)|=|\left(f^{(r)}(x)-f^{(r)}\left(x_{s}\right)\right)+\left(\tau_{1, n}^{(r)}\left(x_{s}\right)-\tau_{1, n}^{(r)}(x)\right) \mid \\
& \leqslant\left\|f^{(r+1)}\right\|\left|x-x_{s}\right|+\left\|\tau_{1, n}^{(r+1)}\right\|\left|x-x_{s}\right| \\
& \leqslant \frac{\pi}{2}\left|\sin \frac{x-x_{s}}{2}\right|\left(\left\|f^{(r+1)}\right\|+\gamma_{5} n \omega_{k}\left(f^{(r)} ; \frac{1}{n}\right)\right) \leqslant \gamma_{7}\left|\sin \frac{x-x_{s}}{2}\right|\left\|f^{(r+1)}\right\| .
\end{aligned}
$$

Интегрируя это неравенство $r-\nu$ раз от $x_{s}$ до $x$ и учитывая то, что $\tau_{1, n}^{(\nu)}\left(x_{s}\right)=$ $f^{(\nu)}\left(x_{s}\right), 0 \leqslant \nu \leqslant r$, получим

$$
\left|f^{(\nu)}(x)-\tau_{1, n}^{(\nu)}(x)\right| \leqslant \gamma_{8}\left|\sin \frac{x-x_{s}}{2}\right|^{r+1-\nu}\left\|f^{(r+1)}\right\|
$$

Отсюда и из неравенства (16) следует, что (см., кроме того, (7) и (6))

$$
\left|f^{(\nu)}(x)-\tau_{1, n}^{(\nu)}(x)\right| \leqslant \gamma_{9} \min _{s}\left\{\frac{1}{n},\left|\sin \frac{x-x_{s}}{2}\right|\right\}^{r+1-\nu}\left\|f^{(r+1)}\right\| .
$$

С другой стороны, из неравенства (16) следует

$$
\left\|f^{(\nu)}-\tau_{1, n}^{(\nu)}\right\| \leqslant \gamma_{4} \frac{1}{n^{r+k-\nu}}\left\|f^{(r+k)}\right\|, \quad 0 \leqslant \nu \leqslant r
$$

и после применения метода Бернштейна (см., например, [2, п. 4.5.3]) имеем

$$
\left\|f^{(r+1)}-\tau_{1, n}^{(r+1)}\right\| \leqslant \gamma_{10} \frac{1}{n^{k-1}}\left\|f^{(r+k)}\right\|, \quad k \geqslant 2 .
$$

Кроме того, в силу (18) при $\nu \in[0, r]$ получаем

$$
\left|f^{(\nu)}(x)-\tau_{1, n}^{(\nu)}(x)\right| \leqslant \gamma_{11} \frac{1}{n^{k}}\left|\sin \frac{x-x_{s}}{2}\right|^{r-\nu}\left\|f^{(r+k)}\right\|
$$

и после применения формулы Тейлора при $k \geqslant 2$ и $k=1$ (см. (20)) имеем

$$
\begin{aligned}
\left|f^{(\nu)}(x)-\tau_{1, n}^{(\nu)}(x)\right| & \leqslant \frac{1}{(r+1-\nu) !}\left\|f^{(r+1)}-\tau_{1, n}^{(r+1)}\right\|\left|x-x_{s}\right|^{r+1-\nu} \\
& \leqslant \gamma_{12}\left(\frac{1}{n}\right)^{k-1}\left|\sin \frac{x-x_{s}}{2}\right|^{r+1-\nu}\left\|f^{(r+k)}\right\| .
\end{aligned}
$$

Из (22) и (23) теперь следует

$$
\left|f^{(\nu)}(x)-\tau_{1, n}^{(\nu)}(x)\right| \leqslant \gamma_{13} \frac{\left\|f^{(r+k)}\right\|}{n^{k-1}} \min _{s}\left\{\frac{1}{n},\left|\sin \frac{x-x_{s}}{2}\right|\right\}^{r+1-\nu} .
$$


Выбирая произвольно $g \in C^{r+k}(\mathbb{T})$, учитывая линейность оператора $\tau_{1, n}(f)$ и объединяя вместе оценки (19) и (24), получаем

$$
\begin{aligned}
& \left|f^{(\nu)}(x)-\tau_{1, n}^{(\nu)}(f ; x)\right|=\left|\left[f^{(\nu)}(x)-g^{(\nu)}(x)-\tau_{1, n}^{(\nu)}(f-g ; x)\right]+\left[g^{(\nu)}(x)-\tau_{1, n}^{(\nu)}(g ; x)\right]\right| \\
& \leqslant \gamma_{14}\left(\min _{s}\left\{\frac{1}{n},\left|\sin \frac{x-x_{s}}{2}\right|\right\}^{r-\nu}\left\|f^{(r)}-g^{(r)}\right\|\right. \\
& \left.\quad+\frac{1}{n^{k-1}} \min _{s}\left\{\frac{1}{n},\left|\sin \frac{x-x_{s}}{2}\right|\right\}^{r+1-\nu}\left\|g^{(r+k)}\right\|\right) \\
& =\gamma_{14} \min _{s}\left\{\frac{1}{n},\left|\sin \frac{x-x_{s}}{2}\right|\right\}^{r-\nu}\left(\left\|f^{(r)}-g^{(r)}\right\|\right. \\
& \left.+\frac{1}{n^{k-1}} \min _{s}\left\{\frac{1}{n},\left|\sin \frac{x-x_{s}}{2}\right|\right\}\left\|g^{(r+k)}\right\|\right) .
\end{aligned}
$$

Однако известно (см., например, [15], [2]), что при $h>0$ имеет место неравенство

$$
\inf _{g \in C^{k}(\mathbb{T})}\left\{\|f-g\|+h^{k}\left\|g^{(k)}\right\|\right\} \leqslant \gamma(k) \omega_{k}(f ; h)
$$

(в качестве $g$ можно взять функцию типа Стеклова или просто полином $\tau_{n}$ из неравенства (5) при $n=\left[\frac{1}{h}\right]$, как это видно из леммы 6, например, при $\left.\varepsilon_{n}(f)=\omega_{k}\left(f ; \frac{1}{n}\right), r=k, s=0\right)$.

Таким образом, минимизируя по $g \in C^{r+k}(\mathbb{T})$, при $\nu \in[0, r]$ получим

$$
\begin{aligned}
& \left|f^{(\nu)}(x)-\tau_{1, n}^{(\nu)}(x)\right| \leqslant \gamma_{15} \min _{s}\left\{\frac{1}{n},\left|\sin \frac{x-x_{s}}{2}\right|\right\}^{r-\nu} \\
& \quad \times \omega_{k}\left(f^{(r)} ; \frac{1}{n^{\frac{k-1}{k}}} \min _{s}\left\{\frac{1}{n},\left|\sin \frac{x-x_{s}}{2}\right|\right\}^{\frac{1}{k}}\right) .
\end{aligned}
$$

Это и есть искомая оценка приближения при $n \geqslant N=N(r, k, \Pi)$. При $\left[\frac{m(r+1)}{2}\right] \leqslant n \leqslant N$ можно взять просто эрмитов интерполяционный полином функции $f$ (см. лемму 5 и лемму $12, \mathrm{~b})$ ).

Для доказательства последнего утверждения в теореме (о показателе $\frac{1}{k}$ ) применяем лемму 9. Неравенство

$$
\left\|f-\tau_{n}\right\| \leqslant \gamma(\alpha) \omega_{k}\left(f ;\left|x-x_{0}\right|^{\alpha}\right)
$$

не верно ни при одном $n$, если $\alpha>\frac{1}{k}$. Действительно, в противном случае для всех $f \in C^{k}(\mathbb{T})$ имеем

$$
\left\|f-\tau_{n}\right\| \leqslant \gamma(\alpha) 2^{k}\|f\|, \quad\left\|f-\tau_{n}\right\| \leqslant \gamma(\alpha)\left\|f^{(k)}\right\|\left|x-x_{0}\right|^{\alpha k} .
$$

Из второго неравенства при $\alpha>\frac{1}{k}$ следует, что $\tau_{n}\left(x_{0}\right)=f\left(x_{0}\right)$, а после деления на $\left|x-x_{0}\right|$ и перехода к пределу при $x \rightarrow x_{0}$ получаем $\tau_{n}^{\prime}\left(x_{0}\right)=f^{\prime}\left(x_{0}\right)$. Это противоречит лемме 9 .

ДокАЗАтЕЛьСтво теоремы 3. Утверждение а) доказано в лемме 4.

Докажем утверждение b). Если $\left\|f-\tau_{n}\right\|=E_{n}^{\mathbb{T}}(f)$, то в силу утверждения а) при любом $\varepsilon>0$ можно построить полином $\tau_{f, n}$ такой, что $(r=0)$

$$
\tau_{f, n}\left(x_{s}\right)=f\left(x_{s}\right)-\tau_{n}\left(x_{s}\right), \quad 1 \leqslant s \leqslant m, \quad\left\|\tau_{f, n}\right\| \leqslant(1+\varepsilon) E_{n}^{\mathbb{T}}(f) .
$$


Полагаем $\tau_{1, n}:=\tau_{n}+\tau_{f, n}$. Тогда $\tau_{1, n}\left(x_{s}\right)=f\left(x_{s}\right), 1 \leqslant s \leqslant m$ и и

$$
\left\|f-\tau_{1, n}\right\| \leqslant\left\|f-\tau_{n}\right\|+\left\|\tau_{f, n}\right\| \leqslant(2+\varepsilon) E_{n}^{\mathbb{T}}(f) .
$$

Предположим, что множитель $(2+\varepsilon)$ можно заменить (хотя бы при одном $n$ и одном узле $\left.x_{1}\right)$ на $(2-\varepsilon)$ при некотором $\varepsilon>0$.

Возьмем какую-либо функцию $f_{\delta}$, имеющую на периоде $2 n+2$ точек чебышевского альтернанса, две из которых $x_{1}$ и $x_{1}+\delta$ соседние, а $\left\|f_{\delta}\right\|$ не зависит от $\delta$. Можно взять просто кусочно линейную непрерывную и периодическую функцию. Тогда существует полином $\tau_{\delta, n}$ такой, что $\tau_{\delta, n}\left(x_{1}\right)=f_{\delta}\left(x_{1}\right)$ и

$$
\left|f_{\delta}(x)-\tau_{\delta, n}(x)\right| \leqslant(2-\varepsilon) E_{n}^{\mathbb{T}}\left(f_{\delta}\right)=(2-\varepsilon)\left\|f_{\delta}\right\| .
$$

Очевидно, что

$$
\left|\tau_{\delta, n}\left(x_{1}\right)-\tau_{\delta, n}\left(x_{1}+\delta\right)\right| \leqslant\left\|\tau_{\delta, n}^{\prime}\right\| \delta \leqslant n\left\|\tau_{\delta, n}\right\| \delta \leqslant n(3-\varepsilon)\left\|f_{\delta}\right\| \delta .
$$

Поэтому имеем

$$
\begin{aligned}
2\left\|f_{\delta}\right\| & =\left|f_{\delta}\left(x_{1}\right)-f_{\delta}\left(x_{1}+\delta\right)\right| \leqslant\left|\tau_{\delta, n}\left(x_{1}\right)-\tau_{\delta, n}\left(x_{1}+\delta\right)\right|+\left|\tau_{\delta, n}\left(x_{1}+\delta\right)-f_{\delta}\left(x_{1}+\delta\right)\right| \\
& \leqslant n(3-\varepsilon)\left\|f_{\delta}\right\| \delta+(2-\varepsilon)\left\|f_{\delta}\right\| .
\end{aligned}
$$

При достаточно малом $\delta$ имеем противоречие.

ДОКАЗАТЕЛЬСТВО ТЕОРЕМЫ 4 . В силу теоремы 2 при $r=1, \nu=0$ и $n \geqslant \frac{m}{2}$ существует полином $\tau_{n}$ такой, что на отрезке $\left[x_{s}, x_{s+1}\right], s \in[1, m]$, выполнено

$$
-\gamma(r, k, \Pi) \omega_{k}\left(f^{\prime} ; \frac{1}{n}\right) \delta_{n}(x) \leqslant(-1)^{s}\left(f(x)-\tau_{n}(x)\right) \leqslant \gamma(r, k, \Pi) \omega_{k}\left(f^{\prime} ; \frac{1}{n}\right) \delta_{n}(x) .
$$

Если $\tau_{0, n}-$ полином из леммы 10, то положим

$$
\tau_{1, n}(x)=\prod_{k=1}^{m} \tau_{0,\left[\frac{2 n}{m}\right]}\left(\frac{x-x_{k}}{2}\right)
$$

В силу четности $m$ это полином порядка не выше $n$. При $x \in\left(x_{1}, x_{2}\right)$ он меньше нуля, а затем меняет знак в $x_{s}, 2 \leqslant s \leqslant m$. Проверим, что полином

$$
\tau_{2, n}(x)=\tau_{n}(x)-\gamma_{0} \tau_{1, n}(x)
$$

при специально выбранном числе $\gamma_{0}$ является искомым.

При $n \geqslant m$ имеем $\left[\frac{2 n}{m}\right] \geqslant \frac{n}{m}$. Поэтому на отрезке $\left[x_{s}, x_{s+1}\right]$ в силу леммы 10 получаем

$$
\prod_{k=1}^{m} \min _{k}\left\{1, \frac{n}{m}\left|\sin \frac{x-x_{k}}{2}\right|\right\} \leqslant(-1)^{s} \tau_{1, n}(x) \leqslant c^{m} \prod_{k=1}^{m} \min _{k}\left\{1, \frac{2 n}{m}\left|\sin \frac{x-x_{k}}{2}\right|\right\} .
$$

Однако правая часть этого неравенства не более

$$
c^{m} \min _{k}\left\{1, n\left|\sin \frac{x-x_{k}}{2}\right|\right\}=c^{m} \delta_{n}(x) .
$$


Подставим и в левую часть подобную функцию. Считая для определенности, что $x \in\left[x_{s}, \frac{x_{s}+x_{s+1}}{2}\right]$, получаем

$$
\begin{gathered}
n \delta_{n}(x) \leqslant m \min _{s}\left\{1, \frac{n}{m}\left|\sin \frac{x-x_{s}}{2}\right|\right\}, \\
1 \leqslant \gamma_{1}(\Pi)\left|\sin \frac{x-x_{k}}{2}\right|, \quad 1 \leqslant k \leqslant m, \quad k \neq s .
\end{gathered}
$$

Следовательно, при $n \geqslant N=N(\Pi)$ имеем

$$
\min _{k}\left\{1, \frac{n}{m}\left|\sin \frac{x-x_{k}}{2}\right|, k \neq s\right\}=1 .
$$

Поэтому при $n \geqslant N$ получаем

$$
n \delta_{n}(x) \leqslant m \prod_{k=1}^{m} \min _{s}\left\{1, \frac{n}{m}\left|\sin \frac{x-x_{k}}{2}\right|\right\} \leqslant m(-1)^{s} \tau_{1, n}(x) .
$$

Осталось выбрать $\gamma_{0}=\frac{m}{n} \omega_{k}\left(f^{\prime} ; \frac{1}{n}\right)(\gamma(r, k, \Pi)+1)$.

Действительно, при $x \in\left[x_{s}, \frac{x_{s}+x_{s+1}}{2}\right]$ имеем следующую оценку приближения снизу:

$$
\begin{aligned}
(-1)^{s} & \left(f(x)-\tau_{2, n}(x)\right)=(-1)^{s}\left(f(x)-\tau_{n}(x)\right)+\gamma_{0}(-1)^{s} \tau_{1, n}(x) \\
& \geqslant-\gamma \delta_{n}(x) \omega_{k}\left(f^{\prime} ; \frac{1}{n}\right)+\gamma_{0} \frac{n}{m} \delta_{n}(x)=\delta_{n}(x) \omega_{k}\left(f^{\prime} ; \frac{1}{n}\right), \quad n \geqslant N,
\end{aligned}
$$

и подобную оценку сверху.

При $\frac{m}{2} \leqslant n \leqslant N$ для доказательства теоремы подходит полином из леммы $11, \mathrm{~b})$.

Одинаковые двусторонние оценки (сверху и снизу) приближения функций линейными операторами через модули гладкости получены впервые автором (см., например, [1, гл. 5, п. 5] или [2, п. 8.2]). Однако с модулем гладкости производной функции для методов суммирования, построенных введением множителей в ряд Фурье, такие неравенства невозможны. Здесь (в теореме 4) существенно, что операторы $\tau_{n}$ нелинейные.

\section{Список литературы}

1. В.К. Дзядык, Введение в теорию равномерного приближения функиий полиномами, Наука, М., 1977.

2. R. M. Trigub, E. S. Bellinsky, Fourier analysis and approximation of functions, Kluwer Acad. Publ., Dordrecht, 2004.

3. Р.М. Тригуб, "Общая прямая теорема о приближении функций из класса $C^{r}$ алгебраическими полиномами с эрмитовской интерполяцией”, Докл. PAH, 386:5 (2002), 599-601; англ. пер.: R. M. Trigub, "A general direct theorem on approximation of functions of class $C^{r}$ by algebraic polynomials with Hermitian interpolation", Dokl. Math., 66:2 (2002), 247-249. 
4. Р. М. Тригуб, "Приближение функций полиномами с эрмитовской интерполяцией и ограничениями на коэффициенты", Изв. РАН. Сер. матем., 67:1 (2003), 199-221; англ. пер.: R. M. Trigub, "Approximation of functions by polynomials with Hermitian interpolation and restrictions on the coefficients", Izv. Math., 67:1 (2003), 183-206.

5. I. E. Gopengauz, "Pointwise estimates of the Hermitian interpolation", J. Approx. Theory, 77:1 (1994), 31-41.

6. K. Kopotun, "Simultaneous approximation by algebraic polynomials", Constr. Approx., 12:1 (1996), 67-94.

7. М. Г. Плешаков, П. А. Попов, "Второе неравенство Джексона в знакосохраняющем приближении периодических функций”, Укр. матем. журн., 56:1 (2004), 123-128; англ. пер.: M. G. Pleshakov, P. A. Popov, "Second Jackson inequality in a sign-preserving approximation of periodic functions", Ukrainian Math. J., 56:1 (2004), $153-160$.

8. А. Зигмунд, Тригонометрические ряды, т. 2, Мир, М., 1965; пер. с англ.: A. Zygmund, Trigonometric series, vol. II, Cambridge Univ. Press, New York, 1959.

9. М. А. Субханкулов, Тауберовы теоремы с остатком, Наука, М., 1976.

10. Р. М. Тригуб, "Односторонние и комонотонные приближения алгебраическими полиномами с положительными коэффициентами”, Теория приближений и ее приложения (Киев, Украина, 1999), Ин-т матем. НАН Укр., Киев, 2000, 461-476.

11. Р. М. Тригуб, "Приближение периодических функций тригонометрическими полиномами с эрмитовской интерполяцией и учетом положения точки. Кусочно односторонняя аппроксимация", Доповіді НАН Укр., 5 (2006), 19-22.

12. Bl. Sendov, "The constants of H. Whitney are bounded", C. R. Acad. Bulgare Sci., 38 (1985), 1299-1302.

13. Ф. Хартман, Обыкновенные дифференциальные уравнения, Мир, М., 1970; пер. с англ.: Ph. Hartman, Ordinary differential equations, Wiley, New York-LondonSydney, 1964.

14. Г. Полиа, Г. Сегё, Задачи и теоремы из анализа. Ч. 2. Теория функиий. Распределение нулей. Полиномь. Определители. Теория чисел, Мир, М., 1956; пер. с нем.: G. Pólya, G. Szegö, Aufgaben und Lehrsätze aus der Analysis. Zweiter Band. Funktionentheorie, Nullstellen, Polynome, Determinanten, Zahlentheorie, Julius Springer, Berlin, 1925.

15. Й. Берг, Й. Лефстрем, Интерполяиионные пространства. Введение, Мир, М., 1980; пер. с англ.: J. Bergh, J. Löfström, Interpolation spaces. An introduction, Grundlehren der Mathematischen Wissenschaften, 223, Springer-Verlag, BerlinHeidelberg-New York, 1976.

P. M. Тригуь (R. M. Trigub)

Донецкий национальный университет, Украина

Поступило в редакцию

E-mail: roald@ukrpost.ua

30.08 .2007 